\title{
Kai Hamdorf/Claes Lernestedt Die Kriminalisierung des Kaufes sexueller Dienste in Schweden
}

Prostitution war stets ein heikles Thema für das Strafrecht. Die Skala des strafrechlichen Umgangs mit der Prostitution reicht vom totalen Verbot bis zur vollständigen Freigabe. Als Erfabrung gilt festzubalten: Je repressiver der Umgang mit dem Problem, desio intensiver die Versuche, das Gewerbe im Gebeimen $z u$ betreiben. In dieser Situation ist der schwedische Gesetzgeber einen neuen Weg gegangen: Er hat den Kauf, niche aber den Verkanf sexueller Dienste unter Strafe gestellt. Der Freier und nicht die Prostituierte stehen nun in Schweden unter Strafandrobung. Demgegenüber vertreten die Autoren die These, wenn man eine Kriminalisierung der Prostitution überhaupt für erforderlich hält, dann sollcen beide Parceien betroffen werden. Besser jedoch seien soziale Maßnabmen zur Lösung der Probleme.

Die Red.

"Indeed, there seems to be an undeniable cendency among legislators to assume that the commercialization of an activity takes it outside the sphere of the principle of liberty and subjects it to public regulations'r.'

\section{Einleitung}

Schweden hat seit jeher sowohl in gesellschafts- als auch in kriminalpolitischen Fragen wenn nicht eine Vorreiterrolle, so doch zumindest eine Sonderposicion eingenommen. Dies gilt sowohl für das Sanktionensystem als auch für gewisse einzelne Delikte: Man denke an die strenge Haltung gegenuber Alkohol und Drogen, besonders im Straßenverkehr, aber auch an Kindesmißbrauch und -pornographie, alles Gebiere die erse kürzlich erhebliche geseczgeberische Akcivirät erfuhren. Seit dcm r. Januar 1999 hat das Land durch das Geserz über das Verbot des Kaufes sexueller Dienste nun auch die relativ einsame Spitzenposition im Kampf gegen die Prostitution ${ }^{3}$ übernommen.

Die Prostitution ist sicher kein wünschenswertes, zugleich aber auch ein ebenso sicher nicht gänzlicb verhinderbares Phänomen. Inwieweir man hierauf mit Strafrecht reagieren sollte und welche Beteiligten dabei zu strafen sind, ist seit jeher umstrittent. Die Motive für die jeweils gewählce Lösung variierten und wurden oftrmals nur sehr

\footnotetext{
* Für Simone Gérard, ohne deren Gastfreundschaft und Kochkunst der Arsikel in dieser Fonn niche zustandegekommen wärc.

1 Feinberg, Harmaless wrongdoing, 1990, S.221.

2 Lag am förbud mos köp av sexnella tjänsicy SFS $1998: 408$.

3 Derinden schwedischen Gutachten zur Prossitution steks verwendetc Ausdruck kónshandel (Geschlechushandel) bringe demgegenüber bereits eine gewissc Bewerning zum Ausdruck.

4 In Deutschland wird der Freier derzeit allerdings niche belangt, auch wenn cine Teilnahmesuratbarkeit und auch cinc Ordnungswidrigkeit in engen Grenzen durchaus in Bctrachr kame. Hierzu Gradmann-Schecrer, Die Privilegierung des Freiers im Strai- und Ordnungswidrigkeirenrechr, GA 1999, S. $349 \mathrm{ff}$.
} 
vage ausgedrückt, dies gilt auch für die neue schwedische Regelung. Dem Betrachcer werden eine Reihe einzelner negativer Effekte dargeboren, doch bleiben viele Aspekte ausgeblendet, die bei einer Neukriminalisierung gemeinhin für bedeutend gehalten werden. Nicht alle sollen im folgenden aufgegriłfen werden: Die mögliche Effektivität der neuen Regelung wurde bereits im Geseczgebungsverfaliren durch Praktiker in Frage gestellt,' und die bisherigen Erfahrungen der Polizei und der Gerichte liefern ein eindeutiges Fazit'. Ebenso ausgeblendet bleibu die Grenze der Strafbarkeit: Was ist eigenclich unter Prostitution zu verstehen?' Der Darstellung im folgenden liegen gedanklich Siruationen zugrunde, bei denen es sich eindeutig um Prostitucion handelt und die insofern typisiert sind, als der Käufer ein Mann und die Verkäuferin eine Frau ist ${ }^{\S}$. Der Artikel wird sich nach einem historischen Überblick über die Encwicklung in Deurschland und Schweden vielmehr ideologischen Fragen widmen, vor allem der Frage nach der Berecheigung einer solchen Regelung, wie sie in Schweden Gesetz geworden ist. Auf der einen Scite soll das Strafrecht als letztes Mittel, als ultima ratio der geselischafulichen Reaktion eingesetzt werden, auf der anderen Seite haben wir heure bereits einen Zustand der Überkriminalisierung, of angesprochen als strafrechtiche Inflation«. Daher ist bei Neukriminalisierungen grundsätzlich Restriktivität angezeigt und sollten daran hohe Anforderungen geknüpfr werden. Gleiche Anforderungen haben aber im Prinzip auch bescehende Tarbestände zu erfüllen, die Aufhebung einer insofern nicht (mehr) tragbaren Norm wäre kein Eingestehen von Schwäche, auch wenn es in Zeiten des ^geting tough - populistischer Kriminalpolitik und symbolischen Strafrechrs - als solches gedeutet werden mag. Aus diesem Grunde sind die hier aufgeworfenen Fragen auch für den deusschen Leser von Bedeurung, haben doch gerade im Bereich der Straftaten gegen die sexuelle Selbscbestimmung noch Vorschriften Bestand, deren Legitimität vielerorts bestritten wurde und wird?

S Siehe hierzu sovvie such als Darsecliung der neuen Vorschrifi Cormels, Schweden in Kampi gegen dic Prostitution, Neuc Kriminalpolitik 1999, S. sf.

6 -Mehr sichtbare Polizisten auf dem Straßenstrich sollen die Sexkaufer in Malmö und Görcborg abschrekken. Dic Siraßenprostitution in den Städten nimml zu, obwohl der Sexkzuf zum Jahreswechsel verboten murde. - Dic Straßenprostitution ist last im selben Umlang wic vor der Kriminalisicrung zuneckgekehn, und die Sexkauler sind nicht cinmal mehr besonders vorsichtıg. Das Gesetz isz völlig wirkungslose sagr Mikacl Borgave, Sozialarbeiter in Malmös Prostitutionsgruppc. (Aus Dagens Sucrige Nyıt vom 3. 9. 1999).

7 Eine Fragc, die beide Länder gleichermaßen becriff, vgl. LK-Linffhutce, SrGB 11 . Auf. \$180a Rdn 4 (*Verhaltin von Männern und Frauen, die sich einem individuell niche bestimmen Kreis von Mannern und Frauen ze: Sexualkontaken preisgeben oder preisgeben wollerux); zur Diskussion der Definitionsfragen siche Traskman, Gär det att tygla lustan?, JFT 1998. S. 359 ff. Traskman meine, daß dic in der Regei aufgezählıen Merkmale alle vage sind: a) Kommerzialita, b) Promiskuität, c) die Wahl der Geschlechispartner geschicht aus rein wirtschaftlichen Gründen, d) dic sexuelle Vexbindung ist vorübergehend und kurzwährend, sowic e) geprägt durch emotionelle Glcichgültigkeit.

s Daher wird im folgenden von "der Prostituierten- und idem Freter" die Rede scin. Die meiseen der Errvagungen ließen sich aber unproblematusch auf andere hetero- oder homosexuelle Konstellationen überragen, die alle von der envälenten Definition criaßt werden.

9 Vgl. vor allem schon Lauemam, Scxualdelike - Strafuaten ohne Opferz', ZRP 1980, S. 44 ff: .... das geltende Strafreche schützt, unter Miß́brauch des Freihcisbegriffs, die Sexualmoral, «; aus jüncrer Zcil aber duch Gle $\beta$, Die Reglementicrung der Prostiturion in Deueschtand, Diss. 199\%; Gradmann.Scheener (Fn. 4), S. 359 . 


\section{I. Deutschland ${ }^{10}$}

Vom I 8. Jahrhunderc an waren Prostitution und/oder Kuppelei zwar verboten, gab es aber stets in schwankender Zahl - je nach Durclisetzungsfähigkeit der Ministerien gegenüber der Polizei - polizeilich konzessionicrte Bordelle. Durch die mit den Konzessionen verbundenen Auflagen wurden gewisse Hygienestandards durchgesetzr, aber auch besonders negarive Phänomene der Straßenprostitution, wie z. B. Gewalt der Freier gegenüber der Prostituiercen, in gewissem Maße unterbunden. Auf diese Weise konnte man zwei Fliegen mit einer Klappe schlagen: Einerscits wurde die Borschafr eines strafrechrlichen Verbotes - daß die (anständige bürgerliche) Gesellschaft Abscand von Prosciturion und Kuppelei als unakzeptablen Phánomenen nimmt - aufrechterhalten, andererseits aber auch die negativen praktischen Auswirkungen der Prostitution bekämpft und das Milieu überwacht und kontrolliert, was richt möglich gewesen wäre, wenn man es durch strikte Gesetzesanwendung in den Untergrund getrieben härce. Im Aligemeinen Preußischen Landrechr von 1794 komme diese Doppelzüngigkeit direkt im Gesetz zum Ausdruck. In dem nicht weniger als 27 Paragraphen umfassenden Absclunitc "Gemeine Huhrereix wurde sowohl ein generelles Verbot der Prostitution als auch eine Art Rahmenregelung für die polizeilichen Bordellkonzessionen aufgenommen". Zulässige Konzessionsauflagen warcn unter anderem ärztliche Zwangsuntersuchungen und Hausdurchsuchungen ohne konkreten Anlaß.

Das Strafgeserzbuch für die preußischen Staten von 1851 verbor in $\$ 146$ jede Art von Kuppelei und Bordellberrieb, während die Prosicuierre gemäß $₫$ I 47 nur dann bestraft wurde, wenn sie den polizeilichen Anweisungen nicht Folge leistece. Die Gerichre akzeptierten jedoch weiterhin polizeiliche Bordellkonzessionen, da man die betroffenen Vorschriften nichr in dem Sinne auslegen wollte, daß sie die Prostituierten zur - ebenfalls verborenen, da i.d.R. den polizelichen Anordnungen widersprechenden - Straßenproscitution zwängen. Das Ministerium versuchte wiederum, das Polizeipräsidium per Anweisung zur Schließung aller Bordelle zu zwingen, was aber nur teilweise und zögerlich geschah. Auf der anderen Seire befürworteren einige pragmacisch eingestellte Juristen sogar staarliche Bordelle. Im RStGB I 87 I wurde das Verbot der Kuppelei und des Bordellbetriebs bei gleichzeitiger Zulässigkeic der anweisungsgemäßen Prosciution beibehalten und die anweisungswidrige Proscitution in den Bereich der Übertrerungen verlagert. Angesichrs der emeuren Zunatime der polizeilichen Konzessionierung der Bordelle wurde in der Literatur konstatiert, $\mathrm{da} B$ »auch die Diensthandlungen des Polizcibeamren, der den Dirnen sitrenpolizeiliche Anweisungen erteilt oder diese überwach, als tatbestandsmäßige Teilnahme/ Kuppelei abgeurteilt werden müßten, und bezüglich des Staates heißt es: „..., bei der Gestatcung oder gar Beförderung von Bordellen wird er selbst zum Kuppler und zum Übertreter seiner eigenen Gesctze. ${ }^{\prime 2}$. So entstand gar die paradoxe Situation, daß die Polizei selbst Straßenprostizuierte in Bordelle einwies, eine für sich genommen bereits strafbare Handlung.

10 Siche zur Gesamedarstellung der Entwicklung für Deutschland Gleß (Fn. 9), passim und kürzer dees, Obrigkeil und Hurenwirt. ZRP 1994, S. 436 ff.

1 Womit die Gesetzeslage 1794 weinus cher mit den Anfordernissen der Rechrsstaatlichkeit im Einklang srand als es in den jolgenden 200 Jahren der Fall war. Das Nlodell des generellen Verbots der Prostitution bei gleichxeitio gelenden polizeilıchen Konzessionsregelungen für Bordelle wurde dem Preußischen Bordellreglemene vom 2.2. 1792 entlchm und in ALR in den Absebunt Theil II Tirel $20 \$ \$ 999$ bis 1026 uber $\times$ Gemeine Hulurereis eingefügt.

12 Zitich nach $C l e \beta($ Fr. 10), S. 439 . 
Im Dritten Reich war die Einstellung des Staates zur Prostiturion noch ambivalenter:

Das Phänomen wurde verachter (Hirler widmete der Prostitution und den Geschlechtskrankheiten in "Mein Kampf $\alpha$ ein ganzes Kapitel), gleichzeitig wurde aber die Regelung der Konzessionierung beibehalcen und die Prostitution gar zu staatlichen Zwecken instrumentadisiert, indem Frauen in Konzentrationslagern zum Geschlechtsverkehr mit Soldaten gezwungen und wohl sogar staatliche Bordelle zur Verhinderung des Verkehrs zwischen ausländischen Zwangsarbeitern und deutschen Frauen eingerichter wurden ${ }^{13}$.

In der Bundesrepublik blieb die Rechrslage bis zur Strafrechrsreform der siebziger Jahre unverändert". Der Reform lag allgemein im Bereich der Sexualdelikte das Bestreben zugrunde, deurlich zum Ausdruck zu bringen, daß Aufgabe des Strafrechts nicht dìe Walırung der allgemeinen Situlichkeit, für den Bereich der Sexualdelikte also die Durchserzung der herrschenden Sexualmoral, sondern lediglich die Bestrafung sozialschädlichen Verhaltens ist". Gesetzestechnisch wurde dies durch die Änderung der Titelüberschrift des 13. Abschnirtes des StGB von "Verbrechen und Vergehen gegen die Sitclichkeitu in "Straftacen gegen die sexuelle Selbscbestimmung « vollzogen. Auch wurden exliche Sexualdelikre grundlegend umgestalter. Im Bereich der strafrechtlichen Regelung der Prostiturion tat sich hingegen vergleichsweise wenig. Einer verwalungsrechrlich-pragmatischen Sperrbezirksregelung, nach der die Landesregierung gem. Art. 297 EGStGB Sperrbezirksverordnungen erlassen kann und die Proscituierte bei Verstoß ordnungswidrig handels ( $\$$ r 20 Abs. I Nr. I OW/G), schließen sich strafrechtliche Vorschriften an, deren Berechtigung vielfach bezweifelt wird $^{\prime 6}$. In $\$ 184$ a ScGB werden Verscöße gegen die Sperrbezirksverordnung bei Beharrlichkeit der Prostituierten zum strafrechtlichen Delikt aufgewertet und in $\$ 1846$ StGB die sittlich jugendgefährdende Ausübung der Prosticucion mit Geldstrafe oder Freibeitsstrafe bis zu einem Jahr belegt. Neben den hier nicht weiter interessierenden Straftarbeständen, die eine catsächliche Beeinträchtigung der Handlungs- und Enrscheidungsfreiheic der Prostimierren voraussetzen, wie erwa die Vorschriften über den Menschenhandel, die ausbeuterische Zuhälterei und Prostitucionsfördenung, und diejenigen Verhalrensweisen, die ein Haiten in Abhängigkeit bedeuten, sind mit $\$$ r 80 a Abs. I Nr. 2 und $₫$ r 8 I a Abs. 2 StGB weiterhin Tatbestände in Geltung, bei denen die umschriebenen Handlungen die Willensfreiheit der Prostiuierten nichr beeinträchrigen, die vielmehr allenfalls cin Indiz für das Vorliegen einer die Willensfreiheit becincrächtigenden Sinacion darstellen können. Während die erste der beiden Vorschriften das ehemalige Bordellverbot ndem sachlichen Gehalt nach unverändert '` übernimmt, erfaße die letztere sogar die nicht-dirigierende Zuhälterei, also das gewerbsmäßige Vermictein sexuellen Verkehrs. Dem alten Verbor der Bordelle und der Kuppelei sind nunmehr aber nicht lediglich neue Namen gegeben, sondern auch neue Schurzinceressen bzw. Rechrsgüter zugrunde gelegt worden. Die Verhinderung der Prostitution selbst ist erklärtermaßen nicht mehr Ziel der Vorschriften, vielmehr geht es um den Schutz der Prostiruierten vor Beeinträchtigungen ihrer persönlichen Unabhängigkeit und Freiheir ${ }^{18}$. Da diese

1; Lau Gleß (Fn. 9). S. 90ff, ist dic histonsche Forschung in dicsem Bcreich allerdings noch rceht wenig enrwickeh.

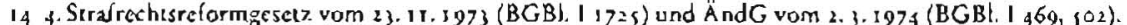

15 BGHSt 23, 43: „Das Strafgesetz liat nicht die Aufgabe, aul geschiechelichem Gcbiet einen moraliseben Standard des erwachsenen Bürgers durchzusetzen, sondern es hat die Sozialordnung der Gemeinschaft vor Storungen und groben Belästigengen zu schutzen..

16 So bezuglich $\$$ is 4 a, b SrGB Leo, Dic strafrechtliche Kontrolle der Prostiution, Diss. 1995, S. 199 , 215 ; Craatmann-Scheever (Fn. i), S. 359: zu $\$ 18$ 3 und zu $\$ \$ 1802$ Abs. I Nr. 2 Alt. 1, i 81 a Abs. 2 SiGB Schönke/Schröder-Lenckner, SiGB, 2 . Aufl. Vor $\$ 174$ Rdn. 2 und insgessme Gieß (Fn. g), S. 1 14 11.

17 Lackner/Kühl, SiGB, 23. Aufl. \$180 a Rdn. 2.

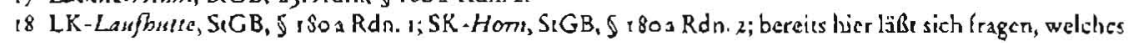


offensichtlich beim bloßen Bordellbetrieb und der entgelrlichen Vermittlung von Kunden nichr gegeben ist, werden die Vorschriften konsequent als abstrakte Gefährdungsdelikte interpretiert's. Die noch im Regierungsentwurl zum sechscen Strafrechtsreformgesetz vorletzren Jahres vorgesehene Umgestaltung des $\$ 180 \mathrm{a}$ Abs. , Nr. 2 StGB in ein konkreces Gefährdungsdelikr ist letzrlich nicht Gesetz geworden:

\subsection{Schweden}

Im Missetacengeserzbuch (misgierningsbalken) des Jahres $1734 \mathrm{kam}$ noch eine äußserst strenge Sexualmoral zum Ausdruck, jeglicher außereheliche geschlechtliche Umgang wurde bestraft und als gemeinsame, gegen Gott gerichtere Sünde angesehen. Im nächsten großen Gesetzeswerk, dem Strafgesetz (strafflagen) von I\$64, hatce sich das Verständnis der Natur der Sexualdelikte bereits in Richrung auf das heute herrschende verändert. Die Beschreibung der Straftaten als gemeinsame Sünde wurde sukzessive vom Bild des Übergriffs eines stärkeren auf einen schwächeren Part abgelöst. So war z. B. der außereheliche Geschlechtsverkehr nicht mehr für sich genommen strafbar. Nunmehr konnte lediglich der Mann bestraft werden, und auch nur dann, wenn der Verkehr zu einer Schwangerschaft führze und er sich seiner Unrerhaltsverpflichrungen enrzog ${ }^{21}$.

Im Jahre 1918 wurde die Strafbarkeir für die verschiedenen Akteure rund um die Prosticuierte wieder verschärft. Danach wurden auch Souteneure bestraft, d. h. Personen, die um des eigenen Gewinns willen dauerhaft die unzüchtige Lebensweise anderer ausnützen, ohne dabei zur fremden Unzuchr zu vermirtein, zu verleiten oder diese zu fördern. Gleichzeitig wurde die Prosriturion selbst, auch im Bordell, entkriminalisiert ${ }^{2 z}$. Die bis dahin bestehende Strafanárohung dürfte hauptsächlich dazu gedient haben, Bordelle als solche zu unterdrücken, so daß mit einer strengeren Strafe für die Umwelt der Prostituierten das Strafbedürfnis hinsichtlich dieser selbst entfiel"'; eine bloß scheinbare Maßnahme, da man Straßen- und Bordellproscituierten mir den scit langem geltenden Landstreichergesetzen beikam ${ }^{24}$. Dabei handelre es sich um rein präventive, nicht strafrechtliche Gesetze, die gewisse spezielle gesellschaftlichc Risikogruppen (wie Betcler, Berulisdicbe, Bauernfänger, Berufs- und Falsch-

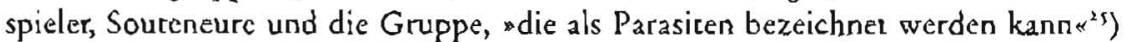
von der Begehung von Straftaten abhaiten sollten. Die Prostituierten konnten so auch nach der Entkriminalisierung mitrels Maßnahmen einschneidender Narur kontrollierr werden. Haupcanliegen scheint auch in Schweden dabei gerade die Kontrolle gewesen zu sein, was durch den Zusammenhang der Landstreichergesetze mir der sog. Reglementierung verdeutlicht wird. Danach wurden die Landstreichergesetze nicht auf diejenigen Prosrituierten angewand, die sich an speziell ausgefertigie Regeln hielten, welche sich auf $\$$ I Landscreichergesecz scürzien. Unter anderem hatten sie sich in polizeiliche Register einzutragen und sich regelmäßig ärztlich

- substantiicrbare Rechesguta - im Untcrsclued zur obloßen Moralwidrigkeit - ciner solchen Unabhängigkeit denn zugrunde licgen soll.

19 LK-Lanflürte, StGB $\$ 180$ a Rdn. 11; Lackner/Kubl, StGB, $\$ 1802$ Rdn. 3.

20 Siche BT.Drs. $13 / 8,87$, S. 6, 32 und BT-Drs. $13 / 9064$, S. 14 .

21 Förslag 1ill Stiaff-Balk, Moliver, S. 38.

22 SFS ig18:459. Und zwar durch Aufhebung des Kap. I 8 in Abs, 2 Simigcset3.

2) Sijernberg. Nils, Koininentar till Straffhger kap. 17-18.2. Nufl. 1930. S. 116-i17.

24 Zur Geschiclice der Landstreichereigesctzgebung siche SOU 1919:4.

2) SOU 1949:4. S. 149. 
untersuchen zu lassen. Kamen sie dem nicht nach, wso wurden sie zu Zwangsarbeit verurteilt, formell wegen gewerbsmäßiger Unzucht, aber in Wirklichkeit wegen Ubertretung des Prostiturionsreglements $\alpha^{26}$. Das Hauprziel der Reglementierung war hier ebenfalls die Kontrolle über die Ausbreitung der Geschlechtskrankheiten. Den regelmäßigen ärzclichen Uncersuchungen folgte bei Feststellung einer Geschlechrsterankheit die Zwangsbehandlung in einem Krankenhaus. Bereits 19r8 wandee man sich jedoch von diesem Reglementierungsmodell ab und versuchce, das Ziel durch die Einführung einer neuen Vorschrift in das Strafgeserz zu erreichen, die die vorsätzliche oder fahrlässige Ansteckung oder Schaffung der Gefahr einer solchen selbst als Delikt ausgestaltere ${ }^{27}$.

In den Vorbereitungsarbeiten der 4 oer Jahre zum heurigen Kriminalgesetzbuch (brottsbalken, BrB) wurde die Wiedereinführung der Strafbarkeit der Prostiturion diskuriert. Unter Hinweis teils auf die gerade vorgenommenen Entkriminalisierungen, unter anderem bezüglich des homosexuellen Verkehrs zwischen Erwachsenen, teils darauf, daß die Prostiturion sich, so gewisse Formen verboten würden, nur wieder andere suche, nahm man von einer Neukriminalisierung Abscand. ${ }^{2}$

Die Frage nach der Ausweitung der Maßnahmen gegen die Prostitucion, darunter die Kriminalisienung der Kunden, der Prosciruierten oder beider, aurde im Prostitutionsgutachten des Jahres 1978 wieder aufgenommen ${ }^{29}$. Hierin wurde empfohlen, weder Verkäufer noch Käufer generell zu kriminalisieren, da man die Prostitution damit lediglich in den Untergrund treibe, mit erhöhtem Risiko für beide Parteien. Es seien auch erhebliche Beweis- und Definitionsschwierigkeiten zu erwarten und die Möglichkeiten der Überwachung der Verbotsbefolgung gering einzuschätzen. Die einzigen Fälle, bei denen es zu einem Eingriff kommen könnte, beträfen die Straßenprostitution, während die versteckte Luxusprostitution unverfolg $t^{3 \circ}$ bliebe, so daß die Kriminalisierung ein Beispiel typischer Klassengesetzgebung darstellen würde. Man sprach sich allerdings zumindest für eine begrenzte Kriminalisierung aus. So solite derjenige wegen sexuellen Mißbrauchs bestrafc werden, der sexuelle Dienste von einer Prostiturienten erwirbt, von der er weiß, daß sie drogenabhägig ist oder unter dem Einfluß von Drogen steht. Dieser Vorschlag wurde jedoch ruchr Gesetz.

Einige Entwicklungstendenzen lassen sich in beiden Ländern gleichermaßen ausmachen. Zum einen ist dies der Widerstreit zwischen pragmatischer Reglementienung - deren rechtsscaacliche Bedenklichkeit auf einem anderen Blact steht - und konsequenter Verdammung der Prostitution und ihres Umfeldes. Zum anderen läßt sich bezüglich der beteiligten Akzeure, Käufer, Verkäufer und Vermittler, folgendes sagen. Als die Zeit vorüber war, in der man die Sexualdelikıe als gemeinsame Sünde beider Parteien angeselen hatte, war es die Schuld des Mannes, die in erster Linie herausgestrichen wurde. Was den Geschlechushandel angehr, war der Käufer seiner norwendigen Rolle zum Trocz nur eine Schattenfigur, während andere Akteure, die die Proscituierte auf die cin oder andere Weise zur Prostinution ermuntern oder Nutzen hieraus ziehen, in weitem Umfang mit Strafe bedroht wurden und werden. Eine Grundannahme nach Überwindung der Sichrweise von der gemeinsamen Sünde

26 Proposition 1918:154, S. 34-35.

27 Gnund hicrür war das Weichen der Überzeugung. daß die positiven sanibaren Effekte die negativen reclulichen und sozialen Aspekte überwogen. Siche proposition $1918: 15_{4}, \mathrm{~S}_{4} 6-47$.

28 "Außerdem wird die Prostitution in großem Umfang in verstecken Formen betricben; um die crlorderliche Effekriviä̈e zu erreichen, ware es daher norwendig, staatlichersents Aufsichtsmaßnahmen in cinem Maße vorzunehmen, das nicht glücklich sein kann und das nichı schecn cher schader als nutzt. Es ist nimlich festzuhalten, daß zumindest was erwachscne Personen betriffe, dic Arr, wie ein Mitburger seine sexuellen Verhälınissc ordneh, prinzipıell einc Sache seincs Privatlebens ist, mit der dic Gesclischaft sich nicht beschäfrigen solltc. "Proposition $1918: 154,5.34-15$

29 SOU 198 r:7r. Prosciturion in Schwoden. Hintorgrund und Gegenmaknahmen.

30 SOU 195 ri: 1 , S. $149-146$. 
scheint auch gewesen zu sein, daß man gegen den Trieb des Mannes nicht viel tun kann oder sollıe' '. Das Bild der Frau, der Verkäuferin im Geschlechtshandel, unterlag einem tiefgreifenden Wandel. Beschreibungen für Prostituierte wic »wollüstig « oder „Freudenmädchen* wurden im Laufe der Encwicklung ersetzt durch Begriffe wie »Opfer" und sausgenurzt*.

\section{Die neue schroedische Regelung}

Der Käufer scheint nun zumindest in Schweden aus dem Schatten hervorzutreten. Seit dem ersten Januar 1999 wird derjenige wegen Kaufs sexueller Dienste mit Geldstrafe oder Freilieitssirafe bis zu sechs Monaten bestrafi, der sich gegen Entgelt eine vorïbergehende sexuelle Verbindung verschaffe, soweit die Tac nicht im $\mathrm{BrB}$ mic Strafe belegt ist ${ }^{32}$. Auch der Versuch ist strafbar ${ }^{33}$. Die Vorschrife wurde (noch) nicht in den $\mathrm{BrB}$ eingearbeicer, aber in der Gesetzessammlung Sveriges Rikes Lag im AnschluB an die Kuppeleibestimmungen plaziert.

Die Kriminalisienung fügt sich in cin Umfeld von Vorschrilen mit - in geborener Kürzc dargestellt - folgendem Aussehen. Wegen Verfübrtung Minderjabriger, Kapitel $6 \Im 10 \mathrm{BrB}$, wird bestraft, wer durch Angebot oder Hingabe einer Enclohnung sich vorübergehenden sexuellen Umgang mil Personen unter i \& Jahre verschaffe oder zu verschaffen versucht. Wegen Kuppelei, Kapitel $6 \$ 8 \mathrm{Br} B$, wird bestraft, wer fördert oder auf ungebuhrliche Weise ausnutzh daß ein anderer vorübergehende sexuclle Verbindungen gegen Entgelt aufnimmt, sowie der Vermieter, der davon Kenntnis hat, daß dic von ihm vermictece Wohnung ganz oder zum rvesentlichen Teil für entgeltliche sexuel\}e Verbindungen benutzt ivird und der nicht das von ithm Erwartbare zur Vertragsbeendigung unternimmt. Wird die Tatigkeit forlgesetzt oder wicderaufgenommen, so rvird der Vermieter als Förderer dieser angeschen. Darüber hinaus kommt eine Strafbarkcir, wic in Deurschland, auch bei Vorschriften in Bcuracht, die kcine dirckte Verbindung zur Prostiuturion haben, aber aus allgemeinen Gründen anwendbar sein kónnen. Hicrzu zählt die Erregıng öffentlichen Argemisses, die sexuelle Belästigrng, sowie die allgemeine Belästigung, die bei storenden oder aufdringlichen Angeboten oder Nachfragen in Betracht komnen ${ }^{34}$. Wegen sexucllen Mißbrauchs, Kapitel $6 \$_{3} \mathrm{BrB}$, wird der sexuelle Ungang mit P'ersonen bestraft, dic aufgrund persönlicher oder situativer Faktoren als ausgenutzt anzusehen sind. Das Verhältnis des letztgenannten Paragraphen zur Sexkaufregelung wird noch angesprochen werden.

\subsection{Gesetzesmaterialien zur neuen Vorschrift}

Das Prosritutionsgucachten des Jahres 1993 schlägt eine Bestrafung sowohl des Käufers als auch des Verkäufers sexueller Dienste vor's. Die Argumente für und gegen eine solche Kriminalisierung sind im großen und ganzen mit denen der Unter-

31 Aus diesem Grunde wurde sogar die statliche Forderung der Prostutution befurwonct, in Form ciner "Absonderung und Vorbehalning ciner Anzahl von Personen weiblichen Geschlechts zum gelegentlichen schncll vorübergehenden... geschlechtlichen Verkehr... ciner gewvissen Sicherung von Ehe, Familic und Gesellschafesordnung willen $\alpha . L$. Wiese, zikient nach Gleß (Fn. 9), S.90 A nm. 69 .

32 Lag (1998:408) om förbud mot kóp av scxuclly tjänster.

33 W/as bei Straftaten mit vergleichbar nicdrigen Strafrahmen äußerse ungewöhnlich ist. Der Gesetzesrat merkı hicrzu an, odaß es im brousbalken keine andere Simftat mit gleich niedrigem Strafralımen gibt, die im Versuchsstadium mit Suafe belegt ist. Allendings gibr es Beispicte außerhalb des brottsbalken, siehe 2. B. $\$ 40$ Fischereigesetx. (1993:787). "Proposicion 1997/98:55, S. 110 . Auch in der Vorschrifr, dic den Sexkaur von Personen uneer 18 Jahren unecr Sirafe srellt, Kapied ro $\$$ ro BrB, gibt es cine Versuchsbestimmung, телn auch matcrieller Art.

34 Kapitel $16 \$ 16$, Kapiti $6 \$ 7$ und Kapite $4 \$$ > BrB.

3) SOU rg9s:rs, Konshandeln. Betankande âV 1993 års proscitutionsurredning. 
suchung von 1978 identisch (wo hingegen eine generelle Kriminalisierung irgendeiner Parei abgelehnt wurde). Beschiuß-und Wirklichkeitsgrundlage sind ebenfalls ungefähr die gleichen. Der entscheidende Unterschied ist lediglich, daß sie nunmehr anders bewertet wurden. Die Untersuchung becont mehr denn je die narmbildende Funktion einer eventuellen Strafbestimmung: Diese soll klarstellen, daß der Geschlechtshandel nicht sozial akzeptabel ist ${ }^{36}$. Auch soll sie die Gleichheic zwischen den Geschlechtern fördern ${ }^{37}$. Sie wird aber auch als wirksames Mittel bei der Bekämpfung der Prostitution angesehen, nämlich durch Abschreckung. ${ }^{8}$ Die Untersuchung nimmt zur Begründung der Bestrafung beider Parteien in gewissem Maße Abstand von dem Bild des Opfers und des Ausmutzenden. Es ist nämlich, so meint man, fraglich, ob nicht auch der Käufer in gewissem Sinne Opfer ist', und außerdem sei die Prostituierte nicht in dem Maße ausgenutzt, wie es für eine Strafbarkeir wegen sexuellen Mißbrauchs Voraussetzung wäre. Ihr Verhalten reiche auch über dic für eine Tat notwendige Teilnahme hinaus. Das Ziel der Kriminalisierung sei auch nicht allein der Schurz der Frau. Eine einseicige Kriminalisierung gefährde vielmehr die normbildende Funkcion:

- Wenn die Gesellschafr durch Normbildung klarstellon will, daß der Geschlechishandel als Verhalten nicht akzeprabel ist, aus Gründen, die sowohl den Einzelnen als auch die Gescllschaft im Ganzen betreffen, erscheint es natürlich, daß dic festgclegte Norm für beide Parteien, Käufer und Verkäufer, gilt. Den großren Effekt wird eine Strafvorschrift auch nur erreichen, wenn sie sich an beide richect. In dem Fall, daß̧ die Serafvorschrift lediglich den Käufer umfaß̧, hatte dies zur Folge, daß das Verhalten der Prostituienen als weniger verwerflich und methr sozial akzcptabel erscheint. $x^{\text {*0 }}$

Der Vorschlag der doppelten Kriminalisierung wurde von so gur wie allen Remissinstanzen" abgewiesen. Drei von ache befürworteten eine ausschließliche Kriminalisierung des Käufers, während fünf auch diese Lösung ablehnten. Interessanterweise finden sich uncer den fünf Instanzen, die auch die einseitige Kriminalisierung ablehrten, die Zentrale Sozialbchörde, die Reichspolizeidirektion, das Zentralamt für Gerichtswesen, der Juscizkanzler und der Rcichsankläger, somit also im Prinzip alle Remissinstanzen mit stark juristischem Bezug. Auch wurde besonders die Gefahr mangelnder Effekcivität betont. In den auf die Untersuchung folgenden Gesetzesvorschlag (proposition) wurde trocz dieses Widerstandes die einseitige Kriminalisierung aufgenommen ${ }^{12}$. Wie wurde dies begründet? Es scheint so, daß man in höherem

36 SOU 1995:15, S. 17. "Meınes Erachtens ist dic Kriminalisicrung des Geschlecheshandels nun ein nocwendiger Sehrit, um unsere Einstellung in dieser Frage zu markicren und klar Stellung zu bexichen gegen cinc Normalisicrung der Prostitution. $\alpha$, S. 224 und "Dic Zcit ist meines Erachtens nun reif für dic Stantsgewalt, den Geschlechtshandel unter Strafe zu stellen und damir klar zu machen, daß̧ es niche hinnchmbar ist, daß gewisse Menschen andere ausnuzzen und ihnen schaden, wm ihren eigenen Sexualtricb zu befriedigen, so wie man es bereits früher getan har, wenn jemand um des finanziellen Gewinnes willen oder aus anderen Gründen die Prostitution gefordert hat.a, ebd.

37 Eine Strabestimmung würde eine normbildende Funktion erfüllen, das Verhälınis zwischen Fraucn und Mannern in Richtung auf mehr Gleichberechrigung becinflussen und klarstellen, daß der Geschlechushandel nicht sorial akzecptabel ist, sondern cine Tatigkeit, dic dic Staatsgewalt als so verwerflich :nd schadlich für dit lnvolvicrien ansich?, daß ste den Stempel der Straftat aufgedrückt bekommt*, SOU s999:15. S. 221 .

38 Zuvorderst der Käulcr, aber auch der Neurckruticrung von Vcrkauferinnen. SOU 1995:15, S. 221.

39 -Es wurde geltend gemacht, dic Frau sei die Ausgenutzte, das :Opfer im Geschlechtshandel, und daß es daher beacheliche Gründe dafür gebe, bei einer Kriminalisierung nur die Handlung des Käufers als strafbas anzuselen ... Man kann allerdings in Frage stellen, ob niche auch Manner, die regelmäßßig sexuelle Dienste kaufen, in gewivssem Sinne Opfer sind. Daß die Frau in höherem Maß̧e als der Mann durch den Geschlıcheshandel geschädigt wird, ist daher kein entscheidender Grund für cine alleinige Bestrafung der Handlung des Mannes. « SOU 1995:19, S.227.

40 SOU ig9s:r9, S. 227.

41 Diejenigen Verbände und Insucutionen, denen im Verlaufe des Gesctzgebungsveríahrens Gelegenheit 7ur Stellungnahme gegeben wird.

42 Proposition 1997/98:5s. "Kvinnofrid.e. 
Maße als in der Untersuchung eine "Ausnutzender/Opfer a-Perspekcive eingenommen hat. So wurde zum Beispiel als ein möglicher Strafzumessungsfaktor das $\bowtie$ Alter des Opfers ${ }^{43}$ angegeben. Auch nahm man eine Art Schätzung vor, wie schädlich die Prosticution ist.

Die Srrafbarkeir der Prostituierren wird einerseits mit folgender Begründung abgelehnr:

-Auch wenn dic Prosticution als solche keine wünschenswerte gesellschaftliche Erschcinung ist, so wäre es doch unbillig, auch denjenigen zu kriminalisiercn, der zumindcst in den meisten Fällen der scliwächere Part ist, der von dem anderen ausgenutzt wird, welcher nur seinen eigenen Sexualtrieb befriedigen will. Es ist auch wichtig, um die Prostituienen zu motivieren, Hilfe zu suchen, um von der Prostirution loszukommen, daß sie sichr das Gefühl haber, irgendeine Form von Sanktion enwarten zu müssen, weil sie als Prostituiene tatig waren. $x^{41}$

Die Kriminalisierung des Käufers wird andererseits wie folgt begründet:

"Es steht hingegen außer Zweifel, daß die Prostiturion ernsthalte Schäden sowohl für den Einzelnen als auch für die Gesellschaft mit sich bringt. Im Umkrcis der Prostituierten ist in der Regel umfangreiche Kriminalität angesicdele, wie z. B. Drogenhandel, Kuppelei, Mißhandlungen usw. Die Prostituierc befinder sich auch im allgemeinen in einer sebr schweren Situation. Es ist daher eine rvicheige Angelcgenheir der Gesellschaft, die Prostitution \%u bekämpfer. Auch wenn sich Argumente gegen die Kriminalisierung des Kaufs sexueller Dienste an(ühren lassen, ist dic Regierung doch der Auffassung, daß die Argumente füx eine Kriminalisierung so schwer wiegen, daß es angezeige ist, nun ein Verbot des Kaufs von vorübergehenden sexuellen Verbindungen einzuführen. Dadurch wird die Einstellung der Gesellschatt in dieser Frage markierr. Durch ein Verbor können auch die Prostitution und ihre schädliclien Wirkungen effektiver bekampft werden, als es mit der bisherigen Arbeic gegen Prosticution erreicht wurde. ${ }^{45}$

Der Geschlechtshandel scheint demnach ein so großes Problem darzustellen, daß es angebrachr ist, den Käufer mic Strafe zu bedrohen, während man andererseits die Prostituierten als Opfer der Prostitution ansitht und nicht mir Strafe beiegen möchte.

\section{Gründe für eine Kriminalisierung der Kunden}

Ob eine solche Siche wirklich konsequent und rational begründbar iş, soll im folgenden untersucht werden. Was taugr also allgemein und insbesondere im Bereich der Sexualscraftaten als Geserzesbegründung, und kann man eine solche im konkreten Fall der Bestrafung der Inanspruchnahme sexueller Dienstleistungen geltend machen?

\section{4.r. Straftaten als Angriff auf Schutzinteressen}

Gemeinhin wird die Aufgabe des Strafrechis und jeder einzelnen scrafrechtichen Vorschrift darin gesehen, gewisse Rechtsgüter zu sclü̈zzen bzw. rechtlich geschürzte Interessen zu wahren. Dabei handeit es sich sters um geselischaftliche Interessen, Einteilungen wie Rechtsgüter des Einzelnen, der Allgemeinheit und des Staates diencn nur dem Hinweis auf den Grad der Abstraktion. Erscheint es insgesamt wünschenswert, Strafrecht restriktiv anzuwenden, so ist linsichtlich jeder einzelnen Vorschrift zu fordern, daß angegeben wird oder wenigstens angegeben werden kann, 
welchen Schutzinteressen sie zu dienen bestimmt ist und wanum diese schürzenswer sind. Insbesondere stellt sich auch die Frage, warum das angegebene rechclich geschützte Interesse schwerer wiegt als der mit jeder Handlungsanweisung verbundene Eingriff in die Handlungs- oder Organisationsfreiheiten der Normadressaten ${ }^{46}$. Hierauf hat in Schweden bereits Joban Thyrén aufmerksam gemacht:

"Olt ist dic Tätigkeir des Gesetzgebers bei der Fixierung des Gesellschaftswillens mehr instinktiv als klar bewußs, was den Grund für seinen Wfillen angehe (das Interesse, das er eigentlich schützen will). Er hat z. B. das Gefühl, daß Meincid [als Delikr] existieren sollte, aber cr hat dieses Gefühl richt analysien und sich nicht klargemachl, welches Inreresse betroffen ist: ob der Meineid kriminalisien wird aus der Perspektive einer Straftar gegen dic Göulichkeit, gegen das allgemeine Vertrauen, oder gegen die richtende Funktion des Staates. Die Frage wird dadurch weizer kompliziert, daß ... reilweise eine Verschiebung ... gerade in der Auffassung über das gescllschafeliche Interesse vor sich gehen kann, während das spezielle Institut in etwa unverändert bestehen bleibr (z. B. verschiedenc Interpretationen der religiösen Delikte zu unterschiedlichen Zeiten, reso. als Angriff auf die Götrlichkcit, auf den Staat oder das religiöse Ge(ühl). Alles dics vereinc mit der fehlenden Klarstellung des eigenclichen Ziels des Geserzgebers für sich selbst, «"

Ein Grund für die historische Ambivalenz des Gesetzgebers im Verhälnnis zur Prosticution könnte in der durch uncerschiedliche politische und religiöse Ideologien hervorgerufenen Unsicherheit darüber liegen, was überhaupt geschützt werden soll und darf. Die Gesetzesvorarbeiten zu der neuen schwedischen Vorschrifo servieren dem Interessierten ein ganzes Buffet angegriffener Interessen und Gründe sowohl für als auch gegen die Neukriminalisierung ${ }^{4}$. Die einseitige Kriminalisierung in Verbindung mit der in dem Gesetzesvorschlag stärker denn je betonten Opferperspektive können so verstanden werden, daß die Tat zum großen Teil als Angriff auf die Prostituierte angesehen wird. Im folgenden soll daher zunächst der Sexkauf als Straftat gegen die Person (d.h. auch, aber eventuell nicht nur gegen deren sexuelle Selbstbestimmung) und im Anschluß als Suraftat gegen allgemeine Interessen untersucht werden.

\subsection{Sexkauf als Siraftal gegen die Person}

Der Geseczgeber sieht die Sexualdelikte heurzurage nichr mehr als gemeinsame Sünde gegen Got, sondern als An- und Übergriff auf die (sexuelle) Integrität anderer oder als Ausnutzung eines anderen. Das Strafrecht dient niche (mehr) der Schaffung, Verstärkung oder Durchsetzung einer bestimmten Sexualmoral49, sondern bekämpft sozialschädliches Verhalten, d.h. setzt voraus, daß sich zu jedem Delikt ein Scbaden - vermeinclich enger, eine Rechtsgutsverletzung - konstruieren läßt. Man sollce jedoch nicht dem Irrglauben verfallen, durch diese Reformulierung des Zwecks und Ziels der jeweiligen Vorschriften sei viel gewonnen. Auch anscheinend an bloßen Moralvorstellungen orientierte Begründungen argumentieren in der Regel mit gewissen Schäden für die Gesellschaft, wodurch die Trennung von

46 (Rollen)Erwarrungen schallen Freihcir, inüem sic bei rollenkoniormen schadlichen Verlialensweisen nicht entäuscht werden, sie schränken Freiheir aber gleichzcitig such cin, indem sie schädliche Folgen von Verhalten aufgrund Rollenerwartungsverstoßes zurechnen.

47 Johan Thyrén, Strafrïltcons allmanna grunder, 1907, S.8.

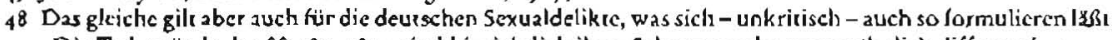
${ }$ Dic Tatbcsiżndc der $\$ 180-181$ a sind hinsichelich ihrer Schwezzwecke ungewóthnlich differenziert...., Lackner/Kuhl, StGB, vor $\$ 174$ Rdn. $\}$.

49 LK-Laufbiule, StGB, Vor $\$ 174$ Abschn. I: s Durch dic Wahl der Abschniusübersehrife hat der Gesetzgeber deutlich gemache daB er sich von überholten Vorstellungen uber das im Zusammenhang mir dem Geschlechtichen strafrechrlich Schüezcnswere lösen wollıe und al's geschützees Rechesgut nicht mehr die Erhaltung allgemeiner Sitclichkcit ansicht.". 
Reche und Moral und die rescrikcive Funktion des Rechtsgutsbegriffs grundlegend in Frage gestellt wird.

Wenn z. B. der Regierungsennvurf 1962 zum Scrafgescizbuch seine ablchnende Haltung gegenuber Enckriminalisicrungsvorscllägen im Bereich der Sexualdelikıe und sogar eine Ausweitung der Strafbarkeiz in Form angeblicher s Lückenschließung a mit der "unbestreirbaren Erkenntnis $\alpha$ begründet, daß die Rcinheit und Gesundheit des Geschlechuslebens eine außcrordentlich wichtige Voraussctzung für den Bestand des Volkes und dic Bewahrung der natürlichen Lebensordnung a sei, dann kann dem nicht mir der Formel des AE begegner werden, das Strafreche habe sich auf die «Pónalisicrung sozialschädlichen Verhaltens« zu beschränken ${ }^{10}$, da ein die Reinlıeiı und Gesundheit des Geschlechtslebens gefährdendes oder gar schädigendes Verhalıen nach ersterer Ansicht eben einen Angriff auf dic natürliche Lebens- und Gesellschaftsordnung darstcllt und solcher in höchsicm Maße sozialschädlich ist. Ebenso licßen sich sowohl Reinheil und Gesundhcit des Geschlechrslebens als aucl die natürliche Lebensordnung als Rechisgüter konstruicren, oline daß man sich dabei sonderlich verbiegen müßte. Auch heute sind ja die "Funklionsfâhigkeie der Rechespflege ader "die Sicherhcit und Zuverlïssigkeit des Rechesverkehrs mit Urkunden a anerkannte Rechesgürtcr. Die entscheidende Fragc ist demnach nichn, ab sich ein Rechtsgut und ob sich Sozialschädlichkeit auffinden Jäßı, sondern warum ein gewisses Verhalten als schädlich anzusehen ist ${ }^{\prime \prime}$. Offensichtlich wird dies bei zirkularen Begründungen, d.h. wenn z.B. bei der Strafvorschrift bezüglich der Mehrehe das Instilut der Einehe als geschützres Rechisgut angegeben wird':

Eine gewisse begrenzende Funktion vermag die Anforderung an Strafvorschriften, sich durch geschützre "subscantiierbare Rechtsgürer und die Angabe der Sozialschädlichkeit des verbotenen Verhaltens legicimieren zu müssen, nur dann zu erfüllen, wenn man den Bereich dessen, was als sozialschädliches Verhalten und als Rechrsgut in Frage kommt, materiell begrenzt. Im Bereich der Sexualdelikre werden dabei der Jugendschutz und der Schutz des sexuellen Selbstbestimmungsrechts genannt, wobei letzreres in Deutschland bereirs in der neuen Abschnitrsüberschrift durch den $\mathrm{Ge}$ seczgeber zum Ausdruck gebrache wurde. Nun fragr sich allerdings, welche begrenzende Funkrion die Angabe dieser Schurzobjekte wirklich auszuüben vermag. Man nehme als Beispiel die Förderung der Prosricucion. Hier sollen auch bescimmte Maßnahmen über das noch Erlaubte hinausgehen, die lediglich "besonders günstige Bedingungen lür die Proscitution schaffen«, wie z. B. das Vorführen pornographischer Filme, die Möglichkeir der Benurzung von Schwimmbad, Sauna und Solarium, sowie Alkoholausschank mit Gewinnbcteiligung für die Prostituierte". Dies ist nur unter der Prämisse verständlich, daß cs sich bei den betreffenden Vorschriften um abstrakce Gefährdungsdelikte im Hinblick auf die Selbsebestimmung der Prostizuierren handele ${ }^{\text {st }}$. Will man hier auch nur eine absrrakte Gefahr sehen, so muß man letztlich davon ausgehen, daß alles, was der Prosticuierten ihre Tärigkeit erleichtert, es

so Bcides ziticr nach LK-Laufhirte, S,GB, Vor $\$ 174$ Abschn. 3.

(1) In diescm Sinnc auch Korialb. Zum Streit um den Begriff des Rechtsguts, GA r999, S. g6, ff, der nachweist, daB nicht dem Begrilf oder der Figur des Rechesgutes irgendeine begrenzende Funkrion zukomme sondern der damil cigenelich gemeineen Forderung nach rationaler Normbegründung (S. 583 ), cine Behauptung, dic sich ganz mit der hicr verresenen Ansicht deckt.

S2 - Geschüzies Rechesgut ist im Anschluß an das in $\$$ S Ehcgesciz enthalicne Verbot der Doppelehe dic auf dem Grundsatz der Einehe beruhende siaaliche Ehcordnurg. * Schönkc/Schröder-Lencerer, SiGB, $\$ 171$ Rdn. r. Vgl. auch Lastmann (Fn. 9), S. 43 zur Straflarkcit der Pornographic: w Wo, beispiclswcisc, läß sich ein Schaden empirisch feststellen, und zwar abseits der fikuiven Sehäden, nach dem Muster des tautologischen A rguments , Devianz verletzt die Norm. (2Pornographie verletze die Sexualverfassung s)? $\alpha$, und S, 48:

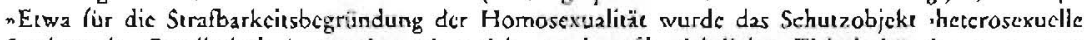
Siruktur der Gesellschafr konstruiert, ohne sich von der offensichtlichen Zirkularitä des Argunents inhibieren zu lassen $\mathrm{Vgl}$. auch Koriach (Fn. 5r), S. 580: ...., streng genommen ist die Existenz enner Ordnung allein kein Grund, sie schon deshalb für schutxiü̈rdiz zu crkliren

S3 LK-Laxßüute, SIGB, $\$$ I 80 a Rdn. I I mir Nachweisen aus der Rechesprechung. Anderc versuchen hicr. restrikriver auszulegen und verlangen Maßnalmen, dic gencrell-abstrake dazu gecignet sind, ctwaigc Abhängigkeiten der Prostuuserten zu vertiefen. Vgl. SK-Hom, StGB, \$180a Rdn. 9; Schönke/SchröderLenckner, StGB, $\$$ r8od Rdr. 13 .

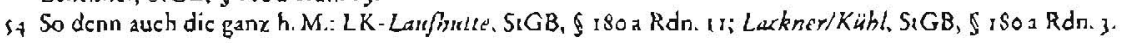


ihr gleichzeitig erschwert, von dieser los zu kommen. Selbst die Werbung für den

Betrieb durch Annoncen stell daher eine verbotene Verhaltensweise dar"

Sicher, Werbung fördert das Geschäfr, dic Kunden mehren sich, die Kasse klingelc.

Doch warum soll bei der Prostitution problematisch sein, was bei anderen gewerbsmäßigen Tätigkeicen nicht nur selbstverständlich, sondern auch erwünscht ist und reilweise staadich gefördert wird? ${ }^{36}$ Oder anders formuliert: Warum ist die Prosticution eben kcin Beruf wie jeder andere? Diese Fragen haben nicht nur politischen und evenuell verfassungsrechelichen Charakter. Ihnen schließt sich nämlich die Frage an, ob und wic Förderung der Prostitution und Sexkauf als Straftaren gegen das Selbstbestimmungsrecht der Prostituierten angesehen werden können." "Die Prostitution ist ... unver einbar mit dem Recht des Individuums, über sich selbst frei zu bestimmen und hindert seine Entwicklung ${ }^{38}$, behauptet ein schwedisches Gutachten. Hier liegt unserer Ansicht nach gerade des Pudels Kern. Soll diesem Satz ein Sinn abgewonnen werden, so muß ihm ein Freiheirsbegriff zugrunde liegen, der nicht rein formalnegativem Muscer folgt, sondern voraussctzc, daß eine substanziell, d. h. wirklich freie Person eine solche Entscheidung nicht träfe. Es ließe sich nämlich auch genau gegensätzlich argumentieren: ‘Soweit eine erwachsene Person auf der Grundlage einer freien Willensentscheidung der Prostitution nachgeht, besteht hierfür kein scrafrechelicher Regelungsbedarf. Der strafrechrliche Schutz von Erwachsenen muß sich vielmehr darauf konzentrieren, die Ausübung von Prostitucion auf der Basis von Gewalt oder Täuschung zu verhindern und die Ausbeurung der Prostiturion zu verfolgenes9.

\section{2. I Negative und positive Freibeit}

Die hier angesprochenen unterschiedlichen Freiheitsbegriffe sind letzelich die von kanrischer Willkür und der hegeischen Freiheic, »ie gerade niche negativ definiert ist, als Freiheit von erwas, sondern positiv, als Freiheit zu erwas, eben zum Allgemeinen, Sitclichen, Guten « ${ }^{60}$.

Nur aus letzterem Freiheitsbegriff ließe sich in zwangsfreier Siruacion eine Unfreiheir der Prostiturierten ableiten und den Kunden, Bordellbetreibern und Zuhältern vorwerfen, sie wirkten daran mic, daß die Prostituierte nicht zur sitclichen Person - im Sinne Hegels, aber eben zugleich auch im umgangsprachlichen Sinne-werden könne. Doch auch Vertreter des formalen Freiheitsbegriffs machen von diesem Ausnahmen in Ansehung bestimmter Situationen. Jobn Stuart Mill meint, daß Eingriffe in Rechte eines Individuums dadurch gerechtfertigr werden können müssen, daß sein Tun anderen schadet. Ansonsten habe die Freiheit vorzugehen, Eine wichuge Ausmahme von dieser Regel macht er jedoch: Dem Individuum kann es nicht erlaubt werden, sich

is Lackner/Kubl, SrGB, $\$ 180$ a Rdn. 4

36 Völfig zutreffend ist daher dic Analyse Homs, SK SIGB, 180 a Rdn. 9: „Dahincer steht offenbar der Gedanke, daß die volle Unabhängigkeir der Prostituierten am besten dadurch erreicht wird, daß sic sich

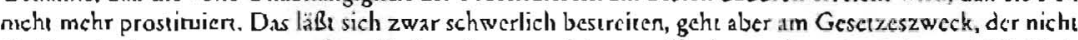
die Prostitution ausrolten, sondern dic Prostituscre schuizen will, aber auch an der Lebenswirklichkeil vorbei, .... .

57 Die ncue Ubersclurift des I 3. Abschnius hat insolern wenig klärende Funktion. Vgl. Lackner/Kubl, StGB, Vor $\$ 174$ Rdn. I: $D$ Daß dic sexuelle Selbstbestimmung geschūtzres Rechtsgut aller Tatbestände de Abschnitus ist, wind durch dessen Überschrif, ... z. war nahagelegt, durch den differenzienen Inhale der Tatbestände und dic Geserzesgeschiche aber nur mit dem Vorbehalt bestätigt, daß der Begriff der Selbsebestimmung sehe weit und allgemein zu verstehen ist... Daß in einzelnen Tatbestanden auch andere Rechtsgiter (mit-) geschùzt sind, sollte dureh dic Uberschrift niche in Frage gesteilt iverden.a.

¿8 SOU igssiss. S. 149.

sq Abrecht st. a., Strafrecht - ultima satio. Empichlungen der Niedersïchsischen Kommission zur Reform des Srrafrechis und des Sirafverfahrensrechts, 1992, S.63.

60 Lesch. Der Verbarcehensbegrilf, 1999. S. 122 
als Sklave zu verkaufen. Dies würde nämlich bedeuten, gerade die Freiheir aufzugeben, auf die man ein Recht hac und die Vorausserzung aller anderen Freiheiten ist:

"The reason for not interfering, unless for the sake of others, with a person's voluntary acts is consideration for his liberry. His voluntary choice is evidence that what he so chooses is desirable, or at least endurable, to him, and his good is on the whole best provided for by allowing him to take his own means of pursuing it. But by selling himself for a slave, he abdicates his libeny; he foregoes any fucurc usc of it beyond that single acc. He therefore deleats, in his own case, the very purpose which is the justification of allowing him to dispose of himself. He is no longer free, but is thenceforth in a position which has no longer the presumption in ils favour that would be afforded by his voluntarily remaining in it. The principle of freedom cannos require that he should be frce not to be free. It is not freedom to be allowed to alienatc his freedom. ${ }^{6 t}$

Wendet man nun die Mill'sche Grundregel und seine Ausnahme auf die Prostitution $\mathrm{an}_{3}$ so ließe sich auf der einen Seire sagen, daß die Freiheir der Parteien beim Geschlechrshandel, wie die aller anderen Individuen, in möglichst geringer Beschränkung durch staatliche Verboce besteht. Das Strafrechc hac sicly nur dann einzumischen, wenn eine Freiheitsausübung für andere Personen von Schaden ist, oder eben zur ^Beschränkung meiner Freiheit oder Willkür, daß sie mit jedermanns Wilikür nach einem allgemeinen Geserze zusammen bestehen könne« im Sinne Kants. Das Strafreche dürfte dann nur bei Zwang und Mißbrauch, nicht aber bei beiderseitig einverständlichem Hande!n in Aktion treten.

Auf der anderen Seire ließe sich jedoch die Proscitution eben als Verkauf seiner selbst in die SkJaverei auffassen. So nehmen sich ja auch seir geraumer Zeit Stellungnahmen

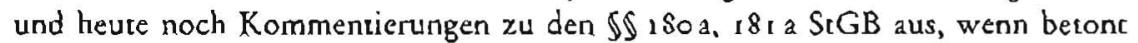
wird, daß es durch »verfestigte Institutionen * im Milieu der Prostitution der Prostivuierten schwerer gemacht werde, sich wieder aus dieser zu lösen. Dahinter stehr ein Bild der Frau, die, einmal ins Milieu abgeglirten, sich aus diesem nichr mehr zu befrcien vermag. Angesichts dieser permanenten und globalen Unfreiheit wird die temporäre und lokale Freiheir der jeweiligen Enrscheidung für einen Freier marginalisiert. Aber auch Kant verdammt die Prostitution:

„Wenn nun aber eine Person sich aus Interesse als cin Gegenstand der Befriedigung der Geschlechremcigung des anderen gebrauchen läßt, wenn sie sich zum Objekı des Verlangens des anderen macht, dann disponierr sie über sich als über eine Sache und macht sich dadurch zu ciner Sache, wodurch der andere seinen Appetic stillt, ebenso wie durch Schweinsbraten seinen Hunger. Nun ist offenbar, da die Neigung des anderen auf das Geschleche und niche auf dic Menschheit geht, daß die Person ihre Menschheit zum Teil dáahingibr und dadurch in Ansehung der moralischen Zwecke Gefahr läuft. Der Mensch ist also nicht befugt, zur Befriedigung der Geschlechecrneigung aus Interesse sich als eine Sache dem anderen zum Gebrauch darzugeben, denn alsdann läuft seine Person, seine Menschheiı Gefahr, von judermann als eine Sachc, als cin Instrument der Befriedigung seiner Neigungen gebraucht zu werden. ... Dieses ist das Schändlichste, sich für Geld dem anderen zur Befriedigung sciner Neigung preiszugcben und seine Person zu vermieten ${ }^{61}$

Hier liegr das Entscheidende zwar nicht darin, daß ich mejne Freiheir dazu benutze, mich ihrer zu begeben, sondern darin, daß ich mich durch ihren Gebrauch entmenschliche. Doch haben beide Sichtweisen eines gemeinsam: An die Stelle des ansonsten vertretenen formell-negativen Freiheirsbegriff wird etwas Materielles gesetzt, das die Prostitution als nichr hinnehmbar erscheinen läßt. Eine solche Vorgehensweise birgr sowohl Vorreile als auch Risiken in sich ${ }^{6}$. Gesellschaftlichen Wertungen bezüglich der Frage, was mit dieser Freiheit vereinbar ist, wird ein recht großer Platz eingeräums, und die Frage kann über den Zwischenschrist des "norma-

61 Mill, On Liberty, 1859, S. 173 .

62 Kant, Eine Vorlesung über Ethik, Ausgabe von 1924, S. 207.

6) $\mathrm{V}$ l Ashworth, Principles of Criminal Law, 3. Aufl. ig99, S. 28 f. uber den modernen Kommunitarismus. 
len* Freiheicsgebrauchs leicht in die Bewertung des "Normalen und Unnormalen* abgleiten. Sowoll der schwedische als auch der deursclie Gesetzgeber legen den strafrechrlichen Bestimmungen rund um die Prostitution zum Teil einen solchen materiellen Freiheitsbegriff im Hinblick auf die Prostizierte und deren Selbstbestimmungsrecht zugrunde. Man meint, die Prostitution sei nicht mit Freiheit vereinbas, da ein Element der Freiheit ist, sich nicht zu prostitujeren. Prostitution ist dann ein Beweis für Unfreiheic, und das sexuelle Selbstbestimmungsrechı wird als sehr wichig angesehen. Das sexuelle Selbstbestimmungsrecht ist so wichtig, daß man manchmal nicht einmal selbst darïber bestimmen darf.

\section{2. 2. Motive für eine Einwilligung in sexuelle Handlungen}

Ein Zirat aus der Stellungnahme des schwedischen Reichsanklägers zum jüngeren Prosticutionsgutachten:

"Der Verkäufer befindes sich nicht auf gleichberechtigem Niveau nit dem Käufer und würde selbscredend grundsüızlich niemals eine sexuelle Bezichung zu dem Käufer aufgenommen haben, wenn sie oder er frei hä̈ı wählen können. Auf cine Weise kann man daher den Geschlechtshandel als eine Straftat gemäß Kapitel 6 brottsbalken ansehen, wo der Z Zwang. zu sexuellem Umgang von der reinen Vergervaligung bis zu einer Sirualion reicht, wo der Käufer die unterlegene Siellung des anderen Parners ausnutzt, sozia), ökonomisch oder persönlich. ${ }^{6_{4}}$

Daß der Verkäufer cinc sexuellc Bezichung mit dem Käufer bei einer Wahl nach nichtpekuniären Kriterien in der Regel nicht akzeptiert hätte, ist klar. Die Frage ist, welche Rolle dies im hiesigen Kontext spielt. Viele Menschen würden ihre beruflichen Tärigkeiren nicht ausüben, wenn sic sfreir häcten wählen können. Das Gesetz verlangt aber aus gutem Grunde gerade nicht, daß man in den sexuellen Umgang einwilligt, weil man selbst das begehre, wünscht oder erstrebt, was das sBesondere und Einzigartige des Sex، ist. Es wird außerdem nicht verlangt, daß die Einwilligung gegeben und der Akt durchgeführt wird, damit der Einwilligende sexuelle Befriedigung erlangt ${ }^{65}$. Unzählige andere Morive sind denkbar: Ich kann mich in sexuellen Beziehungen wiederfinden, weil es mir schwer fällt, nein zu sagen, weil ich mir Ancrkennung erhoffe, weil ich den anderen nicht verletzen, weil ich ihn glücklich oder zufrieden machen will, oder weil ich mir von dem scxuellen Engagement andere Vorteile verspreche, vielleicht sogar wirtschaftlicher Art (z.B. in Form einer Urlaubsreise). Im letzteren Fall kann die Grenze zur Strafbarkeit nunmehr in Schweden berührt werden. Es handelt sich aber hierbei nicht um spezifische, sondern um bloß graduelle Unterschiede im Verhältnis zu den Fällen, in denen der eine Kontrahent sich in einer derart unterlegenen Posirion befindet, daß man annimmt, der Spielraum der Wahlmöglichkeir sei zu gering, um noch von einer Wahlfreiheit sprechen zu können. In diesen Fällen hälı màn es für angemessen, die Strafdrohung greifen zu lassen. Der sexuelle Mißbrauch der $\$ \$$ I $74-174$ C SrGB stellt solche Fälle dar, in Schweden ist aber auch der Fall erfaßt, daß ein Arbeitgeber einen Angestellcen unter der Vorspiegelung zu sexuellem Umgang bringc, er würde anderenfalls seinen Job verlieren ${ }^{66}$. In dem sogenannten "Billy Butt $«-\mathrm{Fall}^{6 \%}$ ging es hingegen um das positive Angebor, gegen

$6_{4}$ Zusammenstellung der Sucllungnahmen zum Gutachten der Prostitutionsuntersuchung durch dis Sozialminisccrium. Dnr S97/8 122/IFO, S.8.

6) Eıwas, was auch mir der älıeren Sexuaimoral kaum zu vereinbaren gewesen wäre und auch heure noch bei switen Teilen der Bcvolkenung - insbesondere naturlich der katholischen - nuf erheblichen Widcrstand stoßen würde.

66 So der maßgebliche Kommentar zu Kapirel $6 \$ 3$ und 4 Abs. 1 ; .Als Beispicl fürden Anwendungsbereich der Vorschrift kann ein Arbcizgeber oder Vorgesetzter genannt werden, der durch schweren Mißbrauch der abhängigen Secllung des. Untergebencn, $x$. B. durch dic Drohung mil Entlassung, diesen zum scxucllen Umgang bringt. Kommentar till Broctsbalkcn dcl I, 7. Aufl. r994, S. 2S5. 
Hingabe eine Anstellung zu verschaffen, und auch in Deutschland sind diejenigen Fälle, in denen bisher die Drohung mit einem Unterlassen als für $\$ 240 \mathrm{StGB}$ in ausreichendem $\mathrm{Ma}$ ße verwerflich angesehen wurde, in erster Linie sexuelle ${ }^{68}$. Hieraus läßst sich folgender Grundsarz ableiten: Man kann und darf ein ganzes Bündel nichtsexkeller Motive für Sex haben, nicht aber wirtschaftliche; man kann und darf unzählige Dienste verkaufen, nicht aber sexuelle.

Hier könnte man fragen: Warum soll der Handel mit sexuelien Diensten verboren sein, während so viele andere Leiscungen frei verkauft und gekauf werden dürfen? Die Antwort ist an diesem Punkt weitaus cinfacher. Diese alleägliche Grundfigur der Verdingung soll eben nicht für sexuelle Dienste gelten, denn hierbei geht es nicht um Arbeit, sondern um Sexualirät. Und sexuelle Befriedigung darf, ebenso wie Liebe, nicht zur Handelsware werden. Promiskuirät ist heutzutage zumindest scrafrechtlich akzeptier, aber sobald die Wahl der Partner aus wirtschafelichen Gründen getroffen wird, hat das Strafrecht einzugreifen, denn Sexualitär soll in größtmöglichem Umfang ein Zweck sein und kein Mittel. Ganz anders sehen hingegen die Hurenbewegung und eine zunehmende Ansicht in der Literatur die Prostitution als Beruf wie jeden anderen und damit auch als grundrechtich geschützr $a^{69}$. Denn die Handelsware ist nicht die Frau selbst, nein nicht einmal ihr Körper, sondern lediglich eine Dienstleiscung ${ }^{70}$ : Danüber, daß Sex keine Handelsware sein sollte, läßt sich möglicherweise Konsens herstellen. Es ist aber eine ganz andere Frage, ob das Strafrecht eingesetzi werden soll, um dies zu unterbinden.

\subsection{Sexkauf als Straftat gegen gesellschaftliche und allgemeine Interessen}

Für eine Konstruksion der entsprechenden Delikte als zumindest reilweiser Angriff auch auf allgemeine Interessen, d.h. auf Universalrechtsgüter, gibe es prakrische Gründe. In diesem Fall löst sich nämlich die Einwilligungsproblemarik auf, da das Delikt Schuczelemente enthält, die nichr zur Disposicion der Prostituierten stehen. Gleichzeitig kann sie aber weiter auch als Opfer angesehen werden, so daß man auch die Teilnahmeproblematik umgeht, die aufträte, wenn das Delikt nicht mehr als Angriff auf die Person der Prostituierten angesehen würde". Durch den Einbezug mehrerer Schutzobjekte läßr sich also genau das erreichen, was erwünscht isc: die Strafbarkeit aller Beteiligten außer der Prostituierten selbst. Denkbare und auch teils angeführte derartige allgemeine Interessen sind: der Wunsch, öffentliche Störungen

67 OLG fur Minelschweden (Svea havräis), Uncil vom 17.11. 1993.

68 So der Fall cincs W/arcnlausdetektivs, der mit der Nicheverhinderung cincr Anzcige drohte, um eine $16-$ Jährige zu sexuellen Handlungen zu veraniassen. BGHSt 31, 199, und der Fall cines zukünlıigen A rbeitgebers, der drohte, scinc bloB mündliche Einstcllungszusage nicht cimxuhalten, BGH NJW 1993, 1807. $69 \mathrm{Vgl}$. Laskowski, Dic Ausubung der Prostitution - Ein verfassungsrechtlich geschützler Beruf im Sinnc des Ar. I2 I GG. Diss. 1997.

70 Nach h. M. Koninu es fur das Merkmal der Prostitution nicht aul köperlichen Kontak1 an. Sogar Telefonscx soll darunter fallen konnen, LK-Lawfhïsse, StGB, \$180.a Rdn. 4. Vgl. auch Pateman, The Sexual Contract, 1985: uMany recent feminist discussions have argucd that prostitution is mercly a job of work and the prostitute is a worker. like any other orage labourer... Concractarians anguc that a prostirute contracts out a certain form of labour power for a given period of time in exchange for noney... A prosejuec does not sel) herself, as is commonly alleged, or cven sell her sexual parts, but contraces out usc of sexual services, a ziticn nach Lacey k. a., Rcconstruccing Criminal Law, 1994, S. 358-359. Paleman tcilt jedoch selbse diese Ansiche nicht.

71 Vgl. Jarebong, Bronen 1, 2. Aufl. $19 \$_{4}$, S. 309: $*$ Der Gesetzgeber hat sich entschieden, die Sexualstraftaten als Übergriffe zuf Personen zu konstrvieren, die des Schuizes ihrer sexuellen Integritat bedurfen. Dadurch scluaffe man cin Gegensatzverlältnis zwischen Täıer und Opfer, das niche immer den wirklichen Verhältrissen entspricht. Das Opfer genießt strafrechtlichen Schutz, selbst wenn cs dic T2t gcstattct oder sogar selbst die Initiative dazu crgriffen hat. Aus dieser Konstnktion folgt auch, daß das Opfer nichu wegen Anstiftung oder Bcihilfe bestraft wird.". 
zu verhindern, der Wunsch, das kriminalitärserzeugende oder -fördernde Umfeld der

Proscitution als ganzen Komplex auszurrocknen «, ohne daß der Sexkauf, die Förderung der Prosticution oder die Zuliälterei für sich genommen strafwürdig wären, der Wunsch, der Prostituierten aus der Situation herauszuhelfen, die oftmals der Prosrituierung zugrunde liegr (Mißbrauch, Drogenabhängigkeit u.ä.), ohne daß der Sexkauf, die Förderung der Proscitution oder die Zuhälterei für sich genommen strafwrïrdig wären, und der Wunsch, den Kauf und Verkauf sexueller Verbindungen selbst zu verhindern, zu vermindern oder zumindest moralische Absrandnahme zu markieren, eine Sexualmoral zu schaffen oder zu verstärken.

\section{3. 1. Die Störung für die Allgemeinbeit - privat und öffentlich}

Das schwedische Prostitutionsgurachten beschreibe u. a. auch die Störungen, denen z. B. die Bewohner eines Gebäudes in oder in der Nähe von einem zugelassenen Prostiutionsbezirk ausgesetzr sind. Sie können darin bestehen, daß ein reger Betrieb vorbeifahrender Fahrzeuge mit (potentiellen) Kunden herrscht, oder daß Passanten in der Fehlvorstellung "angegrabscht " werden, sie seien Kunden oder Proscituierte. Dies kann sogar Kindern und Jugendlichen geschehen. Dabei werden auch triviale Unannehmlichkeiten bemerkt: "In Parks und auf Friedhöfen werden Besucher bisweilen unfreiwillige Zeugen der sexuellen Dienscleistungen. Verschmutzungen in Form gebrauchter Kondome und Exkremente an den Orten, wo die Geschlechrsakre ausgeführt wurden, gehören auch zum Gewöhnlichen "s. Die Gutachter kommen allerdings zu der Auffassung, då dies kein Grund für eine Kriminalisierung beider Parteien ist. Im englischen sog. Wolfenden Report ${ }^{73}$ aus den fünfziger Jahren, ideologisch J.S. Mill nahestehend, wird die Grenze zwischen verborenem und erlaubrem Verhalien mit Hilfe des Kriteriums der Oifentlichkeit gezogen. Man ist der Auffassung, daß das, was der Einzelne in sexueller Hinsichs privat und freiwillig tuc, seine Sache ist und die Gesellschaft nichts angeht, und betont dabei nthe importance which society and the law ought to give to individual freedom of choice and action in macters of private moralicy. Unless a deliberace attempt is to be made by sociery, acting through the agency of the law, to equace the sphere of crime with that of sin, there musc remain a realm of private morality and immoralicy which is, in brief and crude terms, not the law's business. ${ }^{7 *}$ Die Funkcion des Strafrechts in bezug auf die Prostitution soll dabei sein, schwache Akteure und die Allgemeinheit zu schützen"s. Man meint, daß die Prostitucion durch das Strafrecht weder ausgerottet werden kann noch sollce, und becons, daß »saubere “ Straßen ein angemessenes und legicimes Ziel seien: "What the law can and should do is to ensure that the streets of London and our big provincial cities should be freed from what is offensive or injurious and made tolerable for the ordinary citizen who lives in them or passes through them. ${ }^{76}$ Ähnlich nimmt sich auch die eigentlich reche pragmacisch angelegte Sperrbezirksregelung in Deutschland aus, die das Verhalten der Prostituierten gemäß $\$$ r84a

72 SOU 1995:19. S. 148.

7) Report of the Commitec on homosexual offenses and prostitution. 1957.

7. Ebd, 61 .

75 .[T] present public order and decency, to proeces the citizen from what is offensive or injurious, and to provide sufficient safeguards against exploitation and corrupion of others, particularly those who are spceially wulnerable because they are young. weak in body or mind, inexpericnced, or in a slatc of spccial physical, official or economic dependence. It is not, in our vicw. the function of the law to intervenc in the pruatc lives of citizens, or to seck to enforec any paricular pancern of behavior, further than is necessary to carry out the purposes we have oullined. Report (Fn. 73), $5 \$ 13-14$.

76 Report (Fn. 73) $\$ 18$ s. Die liberalc Auftcilung in privat und öffentlich wurde von feministischer Seite mchrfach kritisier, da sie z. B. Phanomene wie dic Vengewaltigung in der Ehc niche zu erfassen vermag. Lacey "1, a. (Fn. 70), 5. 315 . 
St GB nur bei beharrlicher Zuwiderhandlung unter Scrafe stellc. Doch wird auch hier bezweifelt, ob ein für Kriminalisierungen hinreichendes Strafunuecht vorliegt, und wurden bereits im AE 1962 die Abschaffung der Vorschrift und verwaltungsrechtliche Alcernativlösungen vorgeschlagen ${ }^{77}$. Erhöhter Fahrzeugverkehr, Parkplatzprobleme und Verschmutzungen scellen - wie ganz zu Recht angemerkt wird - sypische Regelungsmaterien des Bau- und Gewerberechts dar.

Denkbar wäre es aber auch, nicht am Mit-ansehen-müssen der sexuellen Handlungen oder ähnlichem Anstoß zu nehmen, sondern allein daran, daß man weiß, „was da vor sich geht $«$. Hiergegen läßt sich aber folgende Äußerung H. L. A. Harts ins Feld führen:

- The fundamental objection surcly is that a right to be protected from the distress which is inseparable from the bare knowledge that orhers are acting in ways you think wrong, cannor be acknowledged by anyone who recognises individual liberty as a value ... If distress incident to the belief that orhers are doing wrong is harm, so also is the distress incident to the belief that others are doing what you do nor want them to do. To punish people for causing this form of distress would be tantanounz to punishing them simply because others abject to what they do; and the only liberty thar could coexist with this extension of the utilitarian principle is libeny to do those things to which no one seriously objects. $\alpha^{\text {is }}$

Zusammenfassend laßr sich zu diesem Abschnitc festscellen, daß prostitutionsfreie Straßen nicht offiziel\}er Regelungszweck der neuen Kriminalisierung, wohl aber wenn überhaupt - die einzige Wirklichkeicsveränderung darstellen, die überhaupt erreicht wird.

\subsubsection{Das verbrechenserzengende Umfeld und die Situation der Prostituierten}

Diese Motive können aus mehreren Gründen gemeinsam behandelt werden. Der Grund für eine Kriminalisierung ist hier niche der Verkauf oder Kauf eines sexuellen Dienstes selbst. Unter beiden Gesichtspunksen wird eine Veränderung der Wirklichkeit des Geschlechtshandels angestrebt, nicht bloß seiner räumlichen Plazierung, eine Zurückdrängung in den Untergrund zugunsten »sauberer» Scraßen. Es wäre hiernach auch angemessen, beide Partner unter Strafe zu stellen, um größcmögliche Effektivitär hinsichtlich dieser Ziele zu erreichen. Ideologisch sind die beiden Gründe aber von spezifisch unterschiedlichem Interesse. Ist das verbrechensgenerierende Milieu Zielscheibe der Regelungen, so müßte diskutiert werden, inwiefern es zulässig ist, einen eigenen Straftatbestand als Mittel zur Verhinderung oder Verminderung aoderer Straftaten einzuführen ${ }^{\text {79 }}$. Ähniliche Regelungen gibc es bereits, die Strafbarkeit des Eigenbesitzes oder-gebrauchs von Drogen ließe sich unter diesem Blickwinkel betrachten. Gewöhnlicher ist dieser Regelungstyp dort, wo man etwas bekämpfen will, was sich nicht direkt bekämpfen läßt. Das Geldwäschegesetz und ähnliche Tatbestände aus dem Bereich der organisierten Kriminalität sind in beiden Ländern bekannte und diskutierte Beispiele.

77 Altcrnativ. Entwurl cinces Strafgescezbuches, Besonderer Teil, 1968. S. 45. wo betone wird, daß es sich der Art nach um cine ty pische verwairungsrecheliche Regelengsmaecric handelt, da cinc Handlung niche an sich verboten werden soll, sondern lediglich kanalisiert; vgl, auch Harack, Anmerkung zum BayObLG vom \&. I. 197\$, JR 1980, S. 43s und neuestens Gleß (Fn. 9), S. 141 , dic insbesondere dic Gecignecheit der heusigen Losung zum Ausgleich der beciligen lneressen bezwcifcle und eine Lösung mit bauplanungsrechılichem Charaterer vorschläge.

78 Hart, Law, Libcrty and Morality, 1963, S. 461.

79 So explizic LK-Lakfü̈te zur Entsehungsgeschichte des $\$ 181$ त SzGB: DDer im Jahre 1900 geschaffene Tarbestand hazce zum Ziel, den Zuhatier als Schmarotzer der Dirne zu treffen, da das Zuhälıcrtum als Nahrboden fur Verbrechen aller An angesehen wurdc.*. 
Inceressanter isc daher der Einsatz des Strafrechts als Mittel der Eröffnung von Hilfsmöglichkeiten für die Prostiruierte, die sich aus den Mißbrauchsproblemen, lerztlich aber ganz von der Prostiturion lösen können soll. Hier läßt sich festscellen, daß die ratsächlichen Auswirkungen der schwedischen Neuregelung, falls es überhaupr welche geben sollte, negativ sind. Die Argumencation in dem Geserzesvorschlag, zusammen mit dem späteren Gesetz betrachter, führc in Selbstwidersprüche. Diejenigen Prostituierten, die wirklich der Hilfe bedürfen und die catsächlich als durch den Kunden ausgenutzr angesehen werden können - wozu der Geseczgeber allerdings alle Prostituierten rechnet -, sind auch die, die betreffende Probleme haben. Es ist aber gerade diese Gruppe, die sich am ehesten den nunmehr auftretenden Heimlichkeitsbedürfnissen der Kunden beugen und sich mir ihnen der Öffentlichkeit entziehen wird. So werden die Prostituierren in noch stärkerem Maße der nunmehr unsichtbaren Gewalt seitens der Kunden und eventuell anderer Bereiligter ausgesetzt sein. Eher noch könnte eine Bestrafung beider Parteien das angebliche Ziel erfüllen. Die Prostituierte könnte, so sie denn tarsächlich einmal der Beceiligung am Sexkauf überführt würde, einer Betreuung und Berarung zugeführt werden. Dies wäre, wenn es wirklich darum ginge, nicht eine gewisse Verhaltensweise um ihrer selbst willen zu bestrafen, sondern um einer beteiligten Person Hilfe zukommen zu lassen, eher konsequent.

Welche Formen der aufgezeigte Selbstwiderspruch annehmen kann, zeigt die Auslegung des $\$ 180$ a Abs. I Nr. 2 SrGB durch die Rechtsprechung und gängige Kommentare. Alle Verhaltensweisen seitens eines Bordellbetreibers, die der Prostituierten das Leben angenchmer gestalten, sind verboten ${ }^{80}$. Das Motro der Hilfelcistung für die Prostituierte lautet: Hilfe ja, aber nur zur Aufgabe der Tätigkeit als Prostituierte. Solange die Tärigkeit fortgeserzt wird, bekämpft man alle Personen im Umfeld der Prostituierten, die ihr das Verbleiben in der Prosticution erträglicher machen. Ein Zynismus sonder Gleichen ${ }^{81}$. Glaubhafter ist, daß die Betonung der Opfer- und Hilfeperspektive eher auf der zweifelhaften Notwendigkeit besserer Argumente für eine Bestrafung anderer - des Käufers, Bordellbetreibers, Zuhälters - denn auf wirklicher Sympathie für die Prostiruierte beruht.

\subsubsection{Der "Geschlechishandel" an sich}

Bleibr die Frage, welche Interessen Verkäufer und Käufer sexueller Dienste an sich angreifen. Hiermit nähern wir uns dem gesellschafulichen Verständnis des Wesens, der Einzigarrigkeit und des Anwendungsgebieres der Sexualität. Denn in der Frage, ob die Sexualität etwas (auch strafrechtlich) Spezielles ist, dürfte heute weitgehend Eingkeir herrschen. Folgende Äußerungen einer dreiundzwanzig Jahre alten Untersuchung zu den Sexualstraftaten klingen demgemäß heute nahezu radikal: "Angesichts der hier vertretenen Sichr der Sexualstraftaten erscheint es nicht als ausgemacha, daß die Bescimmungen eine eigene Kategorie von Taten bleiben müssen. Dies erweckt nämlich den Eindruck, es gebe eine spezielle sexuelle Integrität, die von jeder anderen Integrität wesensmäßig uncerschieden sei ... Bei einer umfassenderen Revison des BrB kann es sich ... als möglich und angebrachr erweisen, die Sexualstraftaten als eigenständige Kategorie abzuschaffen. « ${ }^{82}$ Träskman $^{8_{3}}$ beschreibr die historisch-ideo-

8o Siche oben Abschniu 4,2.; lür cine restriktiverc Auslegung, Schonke/Schroder-Lenckner, SLGB, $\$ 1802$ Rdn. 13.

8. So auch Lastmarn (Fn-9), S. 4s zu der angesprochener Vorschrif: „... dic Fraucn arbeicen - bei diescm Tatbestand - absolut frciwillig in dem Bordell, und sic finden bel solchem Arrangement ihrar Arbeit metrr Schutz vor Gefahren, als alle juriscische Reglementierung ihnen je zu biecen vermochic. Dic Strafvorschrift crziclt das Gegenteil dessen, was sie mir kaum übersehbarem Zynismus als ilsren Zweck ausgibr.".

82 SOU $1976: 9$, S. $54 \mathrm{f}$

83 Trüskman ( $\mathrm{Fn}, 7)$. 
logischen Interessen, die durch die Kriminalisierung der Pornographie jeweils geschützt werden sollten. In der ersten Hälíte des 19 . Jahrhunderts macht er als Hauptmotiv den "Schutz der Volksseele a zus, womit gemeint ist, daß die herrschende Auffassung über das jeweils Unpassende oder Passende geschürzt werden sollte $e^{\delta_{4}}$. Die Pornographie war aufheizend, was allein schon aus christlicher Sicht der Sexualität verwerflich erschien. Danach übernahm der "Schuzz der individwellen Akteure * die Leitbildfunkcion; man wollte schwache Gruppen (der bei der Pornographie Mirwirkenden) dagegen schützen, sexuell und wirtschaftlich ausgebeucet zu werden. Diese Sichrweise wurde wiederum, insbesondere in der feministischen Theorie, abgelöst vom "Schutz der Frau im Sinne der Weiblichkeit an sich, nicht bloß der jeweils an der pornographischen Darstellung Beteiligten. Die bisher letzte Phase bezeichner Träskman als "Schutz der guten Sexualitätu. Die Pornographie ruft dieser Ansicht nach falsche Verhaltensweisen hervor, die mit der "guten* Sexualität unvereinbar $\sin ^{8}$ s.

Wender man dieses Schema auf die Prosticution an, so entstammen die Argumente auf jeden Fall den drei letzzeren Phasen, auch wenn das Schüzzenswerte (im Hinblick auf die gesetzliche Ausgestaltung) zumeist in den zwei letzten Phasen lokalisiert wird. Dem Schucz der Frauen als Gruppe und ihrer Würde als abstraktem Phänomen scheint heute metr Gewicht beigemessen zu werden als dem der einzeinen Frauen, dem $\bowtie$ Schutz der individuellen Akteure«, hier also der Prostituierten ${ }^{86}$. Erscheint es wie oben gezeigt zwar schwierig, die betreffenden Delikte als Angriffe aut konkrete Personen zu begreifen (insbesondere da nicht allen Prosticuierten dic Rolle eines Opfers zugeschrieben werden kann), so ist es doch einfacher, sie als Angriff auf die • Frau(en) an sich «, als abstrakte Idee, zu sehen. Hierbei handelt es sich nicht um Personen, sondern um ein Phänomen sui generis, daß sich schwerlich in die Form eines Rechtsgures bringen läßs, allenfalls als „Erhaliung der guten Sexualität «, die lecztlich sogar explizit in der Stellungnabme zum neuen Sexkaufsverbot in Schweden angeführt wird: „Die Prostitution vermittelt ein negatives Bild der Sexualität und beeinflußt daher das Verständnis der Sexualität in der Gesellschaft. Daß Frauen bei der Prostitution, Pornographie und in der Werbung ausgestellt und als bloße Objekte behandele werden, als Körper, bringt mit sich, daß die Sexualitär von Gefühlen abgekoppelt wird. Dies wirkt einem Verständnis entgegen, bei dem Sexualität und Gefühle zusammen gehören und wo beide Paruner Verantwortung für einander übernehmen. ${ }^{87}$ Als Schaden für die Gesellschaft im Ganzen werden u. a. Konsequenzen für die Gleichberechrigung der Geschlechter genanne:

"Das bloße Fakrum, daß es cinen Markt gibt, auf dem Männer sich Zugang auf den Körper von Frauen - oder anderen Männern - verschaffen können, um diese für ihre eigenen sexuellen Zwecke auszunutzen, führ zu Konscqucnzen für das Verständnis des Verhältnisses von Mann und Frau. Daß die Fraven wie cine Ware betrachtel werden, die man kaufen oder verkaufen kann, ist unvereinbar mit dem Menschenbild, welches in ciner modernen Gesellschaft vorherr-

$8_{4}$ Zun Zusammenhang von wüberkommenen Volksanschauungene und Rechesguesbegriff siche Konal (Fn. si), S. 579 .

85 Traskman (Fn. 7). S. 362 ff.

86 Bcispicle des Verhälenisses feministischer Theoric ru den Scxualstraftazen, darunter auch der Prosticution, finden sich bei Lacey th a. (Fn. 70), S. 3 s 7 ff; zur Sexualisat übcrhaupi S. jos ff.

87 SOU $1993: 15$. S. 148. Ganz in diesem Sinne auch Dreher in der 33. Auflage des Kommentars zum damaligen $\$ 180$ Rón. I: - Rechisgut ist niche der Verkuppelte selbst, sondern dic Bewahrung des sexucllen Lebens var gefährlichen Einflüssen;... r, wahrend er in der 34. A uflage zum nunmehr unicr neucr, auf dic sexuclle Selbstbestimmung bezogener Uberschnif firmicrenden, im wesentlichen der alien Vorsehrift aber ahnlichen \$ I80a konstacierte: "Rechesgut ist der Schuly vor den mit der Prostieution verbundenen Gefahren fur dic Prostivicre, niche zulctzi für ihre persónlichc Frciheit « (Rdn. I). Wiederum ein Beispiel für cine schlcichende Auspechslung des Rechusgutes bei nabezu glcichbleibender Narm. 
schen solle, wo Frauen und Männer gleıchgestellt sind hinsichelich ihrer Menschenwürde und ihres Rechis auf Integrität und Respekt.. ${ }^{88}$

Das Tadelnswerte scheint dabei nicht in erster Linie ein Angriff auf die weibliche Sexualitär zu sein, sondern die Tatsache, daß der Frau bei der Proscitucion die Rolle zugewiesen wird, männliche Bedurfnisse gegen Entgelı zu befriedigcn. Wenn nun das, was die Prostituierte tuh, vom Kunden als sexuell cmpfunden wird, wird es dadurch automatisch auch zu einer sexuellen Handlung für den Verkäufer? Die Problematik ähnelt der Diskussion um eine hinreichend prazise Definirion der Kinderpornographie; sind alle Bilder, die auf einen Pädophilen sexuell erregend wirken, Kinderpornographie? Bedeutet eine sexuelle Befriedigung beim Käufer, daß auch der Verkäufer durch die und bei der Befriedigung eine Handlung ausführh die mit seiner eigenen Sexualität verbunden ist? Natürlich ist dies normalerweise der Fall, doch keineswegs immer. Wenn der Käufer z. B. sexuelle Befriedigung durch masochistische oder sadomasochistische Behandlungen erlangt, so braucht die Handlung für den Verkäufer nicht einmal objekriv gesehen eine sexuelle zu sein ${ }^{89}$, außer eben in dem Sinne, daß sie für den Käufer einen sexuellen Inhalt hat.

\subsubsection{Geld und Eigeninteresse}

Der letzte der erkennbaren Gründe für eine Kriminalisierung liegt nichs in dem sexuellen Akt oder der Promiskuität selbst. Es ist vielmehr das Geld der Tropten, der das Faß zum Uberlaufen und das Strafreche zum Einsacz bringt. Eine derarrige Ansicht läßt sich sogar mit J. S. Mills Liberalismus vereinbaren. Mill zufolge ist jeder Mensch frei zu run oder zu lassen was er will, so lange sein Verhalcen nicht anderen schadet - neminem laedere. Dies soll unabhängig davon gelren, ob er sich selbsi schader oder sein Verhalten als unmoralisch zu bewerten isc; Mills Ziel ist das Recht der Individuen auf eine autonome Sphäre. Dies soll sogar das Recht anderer Personen bcinhalcen, den innerhalb dieser Sphäre HandeInden zu diesem Verhalcen anzustiften und ihm mit Rat oder Tar beiseite zu stehen ${ }^{90}$. Mill unterscheidet jedoch dasjenige Verhalten, das weder strafbar (schädigend) noch unmoralisch isc, und dasjenige, das zwar nicht strafbar, aber doch als unmoralisch anzusehen ist". Im letzteren Fall ist nach Mill die Möglichkeit eröffnet den Ratgeber zu bestrafen:

"II peoplc must be allowed, in whatcver concerns only themselves, 10 act as seems best to themselves, al thcir own peril, thcy musi equally be free to consult with one another about what is fit to be so done; to exchange opinions, and give and receive suggestions. Whatever is permitted to do, it must be permitued to advise to do. The question is doubtful only when the instigator derives a personal benefit from his advice, when he makes it his occupation, for subsistence or pecuniary gain, to promote what society and the State consider to be an evil ... Fornication, for examplc, must be tolerated, and so must gambling; but should a person be free to be a pimp, or to keep a gambling house? The case is one of those which lie on the exact boundary line becween two principles ... a $n^{91}$

88 SOU 1995.15, S. 148.

89 Das Ummeren, Auspejeschen ader ahnliches sind ja gerade Handlungen, die fur die meisten Becracheer und wohl zuch für dic meister Prostituicrien zunächst keinerlei sexuellen Charakter haben, sondem im Gegentel cher gecigner sind, jede sexuelle Erregung zu bescitigen.

90 - In cases of personal conduce supposed to be blameable, but which respect for liberiy precludes socicty from preventing or punishing because the evil directly resulting falls wholly on the agent; what the agent is frec to do, ought other persons to be equally free to counsel or instigate? a Mill (Fn.61), S. 168.

91 Die heute noch relevante Unterscticidung selbst ist erlicblich àlter, Mill diskutien aber cingehend ihre Berechigung.

$92 \mathrm{Mill}$ (Fn.6r). S. 168\%. 
Mill drückt damit den Gedanken der (später so genannten) "commercialization of vice " aus, daß derjenige Ratgeber, der seinen Rat aus Eigennutz gibr, wie der Zuhälter, Bordellbetreiber oder Veranscalter von Glücksspielen, durchaus stratbar sein kann: Die einzige Person, die von dem eingenommenen Geld der Prostiruierten frei profitieren darf, ist die Prostituicrte selbst. Nicht einmal derjenige, der mic ihr zusammenwohnt und sich von ihr versorgen läß, kann sich der Straffreilyeir sicher sein jedenfalls nicht in Schweden. Zu Recht ließe sich aus dieser Sicht daher fragen, ob nichr auch der Staat lesztlich unmoralisch handelt, wenn er die Einnahmen der Prostituierten besecuert. Handelt er dabei nicht auch als Kuppler, Zuhälter oder zumindest als Soureneur?

Diese Betrachtungsweise scheitert aber an zwei nicht haltbaren oder durchsetzbaren Voraussezzungen. Zum einen stellt sie einen ideologischen Schritt zurück zur Auffassung der Prosticution als gemeinsame Sünde dar, die man bercirs auf den geschichtlichen Müllhaufen entsorgt hatte. Mit religiösem Interesse ließe sich eine solche "Sünde* in einem säkularen Staar kaum begründen. Zum anderen müßte man ja ein gewisses Maß an Tadel gegenüber der Proscituierten aussprechen, was heutzutage politisch schwierig erscheint.

\section{Symbolisches im Strafrecht}

Die Kritik neuer Kriminalisierungen mittels des Begriffs ssymbolischen Strafreches * hat spätestens seit Hassemer Konjunktur. Es bedarf zu diesem Begriff trotz oder gerade wegen seiner häufigen Verwendung im juristischen Diskurs einiger erlăucernder Worte. Entscheidend ist die Trennung zweier Ebenen: die der Funkcion des Strafrechts von der des Inhaltes strafrechtlicher Normen. Zunächss einmal hat das Strafrecht unserer Ansicht nach eine dem Grunde nach rein symbolische Funkrion. Es soll das Festhaltendürfen an normativen Erwartungen crotz kognitiver Enttäuschungen garantieren. Verhindert werden soll, sofern man überhaupt eine Folgenorientierung animms, daß Enträuschre ihre Erwarcungen aufgeben - sich also kognitiv verhairen - und so die Infragestellung der Gelcung der Norm durch die Siraftar perperuieren ${ }^{93}$. Wenn von «symbolischem Scrafrechr«, "Sozialtechnologien oder ähnlichem die Rede ist, dann betrifft diese Kritik beide Ebenen. Zum einen gilt sie einem polizeirechlich-präventiven Verständnis des Strafrechrs, welchem seitens des Gesetzgebers Steuerungsmöglichkeiten zugetraut und abverlang: werden, die dieses nichı zu erfüllen in der Lage ist und in dem alle Kategorien der Zurechnung nur auf Steuerung zukünfrigen Verhaltens ausgerichcet werden, anstatc rückblickend Verantwortlichkeir festzustellen. Hierin liegt eine Verkennung der Aufgabe und eine Überschäczung der Möglichkeiten des Strafrechrs und werden eigendich politikstrategische Ziele verfolgc. Die Frage der jeweils beteiligten Rechtsgüter, Opfer, Schäden usw. liegt nun niche auf der Ebene der Aurgabe oder Funktion des Strafrechts, sondern auf der des Inhalies der Erwartungen, deren Sicherung sich das Strafrecht auf die Fahnen schreibt. Der Inhalt der Erwariungen wird aber von der Politik vorgegeben, und diese hat sehr wohl rational zu begründen, welche inhaltlichen gesellschaftlichen Zwecke sie mit der neuen Regelung verfolgt. Wenn man also Kriminalisierungen in folgender Weise angreift: „Sie [die Reformkonzepte, Anm. der

93 Insofern anders Hassemer. Sozialecchnologie und Moral; Symbole und Rechesgüzer, in: Heike Jung (Hesg.), Keche und Moral: Bciträge 7u sincr Standoribestimmung, 1. Aufl. 199h, der die Aufgabe des Strafrecin in der Formalisicrung sozialer Kantrolle siche. 
Verf.] sind nur scheinbar progressiv, in Wahrheit aber kriminalpolitisch reaktionär, weil sie entweder mit dem (hierfür untauglichen und schädlichen) Mitrel des Strafrechts auch solche Konflikte in intimen Interaktionssystemen lösen wollen, für die die incrasystemacischen Lösungsressourcen ausreichend oder besser geeigner sind, oder weil sie das Strafrecht zu einer folgenlosen feministischen Demonstration mißbrauchen und ein weireres unsinniges Dunkelfeld schaffen. Sie laufen damic auf eine symbolische Gesetzgebung im schlechten Sinne hinaus. $\mathrm{x}^{94}$, so ist damit nicht die immer (auch) symbolische Funkrion des Strafreches gemeint, sondern soll darauf hingewiesen werden, daß der konkrete Inhalt der Norm nicht racional begründet ist, da der Norm keinc Effektivität zukommen wird. Die Frage Koriatbs zur These von der Nowwendigkeit einer Benennung substanriierbarer Rechesgürer im Sexualstrafrechr: „Und wird hier wirklich um Rechrsgüter gestritten oder darum, ob bestimmte Handlungen überhaupt eine kausale (oder andere, vielleicht nur symbolische) Wirkung auf Rechrsgürer entfalten können? " betrifft die andere Seite bloßer Symbolik, nämlich die Frage, ob die verbotene Verhaltensweise übcrhaupc cinen negativen Einfluß auf "das gedeihliche Zusammenleben der Menschen " hat und daher verbietbar ist. Der Vorwurf der bloßen Symbolik bezeichnet also zum einen eine Unangemessenheit des Verbores unschädlichen Verhaltens ${ }^{9 s}$ und zu anderen mangelnde Effekrivität. Lautmann beschreibc in seinem Arrikel "Sexualdeliktc - Straftaten ohne Opfer? s6 $^{96}$ für den Bereich der damals unter neuer Überschrift firmierenden Straftaten gegen die sexuelle Selbscbestimmung das Phänomen bloß symbolischen, eine wie auch immer geartece Veränderung gesellschafelicher Verhälcnisse oder der Situation Einzelner gar nicht mehr anstrebenden, Strafrechts wie folgt:

"Dic Verfolgung der konsensualen Sexualdclikte [und um cin solches handelt cs sich ja bei allen hier thematisierten, Anm. der Verf.] stcht vor begreilichen Schwierigkeiten: weil keine unmittelbar Geschädigten eine Beschwerde führen, muß nach den Delinquenten gesucht iverden. Das besorgt zum cinen die Bcvölkerung durch private Anzeigen, und die Lust dazu wird durch Moralunternchmer... geschürı. Die Eigenaktiviı̈ııen der Polizei können nur sporadisch scin,... Dabei mussen auch fragwürdige Mituel angewandı werden, wic agents provocatcur. Tauschung, Belauschung oder Infiltration. Das Ermessen ist so hoch,... daß die Zugriffe an Willkur grenzen. Andererseits finden Sankrionen bei den meisten Moraldelikten so selten staut, daß die Lerneffekte bei den Bedroluten und Betroffenen nahe Null bleiben."

Dies dürfe den bisherigen Erfolg der Einführung des Deliktes des Sexkaufs in Schweden rechr guc beschreiben". Sicher, dic Zeir, in der man noch im Sinne sozialer Ingenieurskunst glaubte, die Wirklichkeit durch Geserzgebung in kalkulierbarer, genau vorausschbarcr Weise verändern zu wollen, sind vorbei. Nichı zuletzc die Systemtheorie hat dieser Hybris den Boden entzogen. Doch kann diese Sichtweise nunmehr auch nicht durch cine bloße Berufung auf positive Generalprävencion, auf die moralisch-sictenbildende Kraft der Seczung von Symbolen als quasi »volkspädagogischen Mitteilungen ${ }^{\$ \$}$ erserzt werden. Die Borschaft im Bereich der Regelung der Prostiturion lautet, daß derjenige, der sexuelle Dienstleistungen anbieter, stets

94 Schïnemann, Die Mißnchtung der scxucllen Selbstbestimmung des Eheparners als kriminalpolizisches Problem, GA r996. S. 327.

95 Völlig zutseffend dahcr auch Appel, Rechesgüterschutz durch Strafreche'. KritV 1999, 278 ff. und grundlegend Frisch, Wesentliche Strafbarkeitsvoraussctzungen ciner modernen Syrafgesetzgebung, in: Eserl Kaiser/Weigend (Hrsg.), Von totalitärem zu rechisstatlichem Strafrechi, 1993, S. 201 ff., die beide betonen, daßs Rechtsgüter allenfalls mitulbar über primär festzulegende und - auch an der Verfassung und ihrer Grundrechte - zu rechtfertigende Verhaltensnormen gesehüizt werdin. 96 Lawemanm (Fn. 9), S. 8 .

97 S. O. (Fn. 6).

98 Der Ausdruck stammi van Jarebong, Vilkc'n sorts straffrätt vill vi ha?, in: Victor (Hrsg.), Varning för straff. 1994 , S. 32. 
und in allen Situationen Opfer ist (doch ohne daß eine über das Strafrecht weit hinausgehende Hilfe und Unterstüczung gewährc wird) ${ }^{99}$.

\section{Schlußbemerkungen}

Der Mythos der »wollüstigen Huhre ist zum Glück aus dem öffendichen Diskurs verdrängt worden, an seine Stelle ist der der Prostituierten als Opfer getreten. Opfer nichr bloß der gesellschafclichen Verhälınisse, sondern ganz konkret der sie umgebenden und von ihr profitierenden Personen: des Bordellbetreibers, des Zuhälters und des Freiers. Hiergegen isc, was die Mehrzahl der Prosticuierten berriff, nichts einzuwenden, doch welche Konsequenzen hat diese Sichrweise im Zusammenhang mit der geserzlichen Ausgestalung der Regelung der Prostirution?

Sowohl das schwedische als auch das deutsche Strafrecht nehmen sich jeweils nur einzelner Akteure aus dem Bereich der Prostitution an. Man straft die Fördenung des sich prostizuierenden Verhaltens und dieses selbst unter gewissen Umständen, oder eben die Inanspruchnahme sexueller Dienstleisrungen. Zu einem Verbot der Proscitution, der konsequenten Kriminalisierung aller Beteiligten und dem verstärkten Einsacz sozialer Maßnahmen kann man sich genauso wenig durchringen wie zu einer völligen Freigabe, denn zumindest moralischen Absıand möchte man ja markieren. Welche praktischen Wirkungen kasn man von so gearceten Vorschriften erwarten? Sicher, in gewissem Maße wird verdeck, was wir nicht gerne wissen wollen. Auch isc es für das Gewissen angenehm, für das Schicksal der Prosticuierten direkt verantwortliche Personen benennen und den weitergehenden Zurechnungsprozeß abbrechen zu können: „Auf der Ebene der Polirik fungiert der strafjuristische Zugriff auf Systemwidersprüche allerdings als politische gewünschter Ausblendungs- und Verschleierungsmechanismus, durch den gesellschaftliche Probleme personalisier werden, wodurch sie einer politischen Zurechnung entgehen ... Das Herausgreifen von schwarzen Schafen reinige die Herdea ${ }^{i \infty}$.

Die Notwendigkeic, bestehende Geserzgebung durch ein neues Paradigma zu erklären oder neue mic einem alten zu vereinbaren, has in der Strafrechtsgeschichre schon seit jeher teils bizarre Resultate gezeirigr. Heute besteht das Problem darin, daß man in Schweden die Inanspruchnahme sexueller Dienseleiscungen und in Deurschland seit Änderung der Überschrift des I 3 . Abschnitts die Förderung der Prostitution mit dem Paradigma in Einklang gebracht werden müssen, daß die Sexualdelikte Angriffe auf das sexuelle Selbstbestimmungsreche der Prostituierten darstcllen.

Man hat aus gutem Grund die Sichtweise aufgegeben, nach der Sexualdelikre gemeinsame Sünden sind. Für den Handel mit Sex wäre jedoch die Konstruktion als gemeinsame Straftat am ehesten mit dem Ziel zu vereinbaren, dieses Phänomen zu beseirigen, was naheläge, wenn es so unerwünsche ist, nur soll dies eben gerade niche Sinn und Zweck der Vorschriften sein ${ }^{\text {}}{ }^{\prime \prime}$. Dies nicht zuletzt aus dem Grunde, daß sich die Einsicht Bahn gebrochen hat, daß man es nie wird ausrotten können. Der vielleicht ehrlichere Weg ist da noch der französische. Im neuen code pénal lauter die Überschrift des Abschnittes, in dem sich die Strafvorschrifcen über die Zuhälterei finden: "Angriffe auf die Würde der Person « ${ }^{10 x}$. Noch ehrlicher wäre es aber, sich zu

99 Wobci hicr zowischen Schweden und Deuschland insolern differenzict werden muß. als dis nichtstrafreclueliche Untersiützung dort cren größeren Umfang ausmache als hice.

roo P.-A. Albrecht, Das Strafreche in Zugrilf populistischer Politik, SiV 1994, S. $26 \%$.

ror Schonke/Schroder.Lenckner. SiGB, \$180a Rdn. 1 .

ro2 Livre II Chapitre V-I)es autcintes à la dignité de la personre. Die Situalion ist ansonsen der deutscher 
entscheiden, ob man das Problem wirklich strafrechtich angehen will, oder ob man nicht doch eher auf soziale Einsätze setzt. Die konsequente Alternative ist die zwischen der Bestrafung beider Parzeien - des Freiers und der Proscituierten - und der Entkriminalisierung der Prostirurion. Aus Gründen der anzuratenden Zurückhalrung beim Einsatz des Strafreches möchten wir für letzteres plädieren.

Die Wahl zwrischen Moral und Pragmacismus ist zu allen Zeiten schwierig und umstritten gewesen. Ein lehrreiches Beispiel hierfür liefert Gustaf IIl. Der König versuchre, der in der zweiten Hälfte des i 8 . Jahrhunderts stark zunebmenden, durch die Ausstoßung unverheiratecer Mützer aus der Gesellschaft provozierten Kindestö-

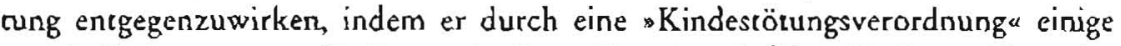
soziale Verbesserungen für Frauen in dieser Situation einführte ${ }^{(0)}$. Er enwiderte der Priesterschaft, die der von ihr so bezeichneten "Hurenverordnung a auf dem Reichstag des Jahres 1786 erbitterten Widerstand leistere:

- Dic Erfahrung har nicht gezeigt, daß durch die frühere Besurafungsweise, die dic Pricsterschaft wiederhergcstells haben will, die Kindestönng in mehr oder weniger großem Umfang aufgehort has, aber wohl, daß eine geschwïngere Frau das Kind als Unglück ansah und Gewalt gegen ein sinnloses Leben anwendete zu iturer cigenen Reztung. Wetche Mictel bleiben da andere übrig, als sic durch einc mildere Behandlung auf den Gedanken zu bringen, daß sie Murter iss, ... nichı sie abcr für ihr ganzes Leben in Unglück und Nor zu sturzen, oder in Verzwciflung, die mit der Verschwendung beider Leben endet, des einen unschuldig, des anderen durch cin Verbrechen. Einrichtungen für den Unterhalt und die Aufziehung des Kindes, an die zugleich gedacht wurde, haben bereits ihre Wirkung gezeigr wo sic begonnen wurden. Sie auszuwciren, dazu bedarf es Zeit und Geldminel. Wenn ein cinziges Leben gerettet werden kann, bewcist dics den Nutzen, wieviel mehr, wenn mehr gerettct werden? Weiter kann man nicht kommen. Die Welt bleibr immer Welt, in der das Schlechte oft vorkommen, nie aber ausgerottet werden kann und wozu sollen sonsı Gesetze nötig scin? «

Bernhard SchlinkRalf Poscher

\section{Der Verfassungskompromiß zum Religionsunterricht}

Für den Konflikt um den Religionsunterricht in den neuen Ländem ist von entscheidender Bedeutung, wie die Bestimmungen des Grundgesetzes zum Religionsunterricht entstanden und gemeint sind. Die Untersuchung zeichnet anhand weitgehend erstmals gesichteter Materialien die einschlägigen Diskussionen des Parlamentarischen Rates nach.

2000, 91 S., brosch., 38,-DM, 277,- oS, 35,50 sFr, ISBN 3-7890-6806-3

\section{NOMOS Verlagsgesellschaft}

76520 Baden-Baden

schrähnlicls: $n$ Les auteurs de l'2cte principal (prostizuć) qui nc sont pas susceptibles d'être poursuivis sont considérés comme des victimes des personnes qui les one aidés à l'accomplir-a Juris-Classeur l'énal j, 1997 Ar. 225-5 ì [2, Introduction.

103 Durch die Verordnung wurde es ihnen u. a. crmöglicht, ihr Kind unter Betreuung in Anonymität und ohne Nennung des Visers zu gebären. Auch war cine genisse Untersivizung fur das Kind vorgesehen. und wurde es der Kirche verboten, die Muter aus der Glaubensgenicinselialt auszuschlies\$en.

104 Wradergegeben in Anners, Humanitet och ratıonalism, 1965, S. 318-319. Die Äußsenng des Königs surde von Reichsral von Hemansson verfaße. 